\title{
Pricing and hedging Asian-style options in energy
}

\author{
Fred Espen Benth* and Nils Detering ${ }^{\ddagger}$
}

August 14, 2014

\begin{abstract}
We solve the problem of pricing and hedging Asian-style options on energy with a quadratic risk criterion when trading in the underlying future is restricted. Liquid trading in the future is only possible up to the start of a so-called delivery period. After the start of the delivery period, the hedge positions can not be adjusted anymore until maturity. This reflects the trading situation at the Nordic energy market Nord Pool for example. We show that there exists a unique solution to this combined continuous-discrete quadratic hedging problem if the future price process is a special semimartingale with bounded mean-variance tradeoff. Additionally, under the assumption that the future price process is a local martingale, the hedge positions before the averaging period are inherited from the market specification without trading restriction. As an application we consider three models and derive their quadratic hedge positions in explicit form, a simple Black Scholes model with time-dependent volatility, the stochastic volatility model of Barndorff-Nielsen and Shephard and an exponential additive model. Based on an exponential spot price model driven by two NIG Lévy processes, we determine an exponential additive model for the future price by moment matching techniques. We calculate hedge positions and determine the quadratic hedge error in a simulation study.
\end{abstract}

JEL Classification: C61, G11, G12, G13

2010 Mathematics Subject Classification: 91G20, 60G99

Keywords: Asian options, energy markets, trading restrictions, quadratic hedging, moment matching

\section{Introduction}

In this paper we address the problem of pricing and hedging Asian-style options on energy when trading in the hedging instrument is restricted in a period preceding maturity. This is motivated by the market situation at the Nord Pool energy exchange, one of the world's largest exchanges for electricity, covering the northern European region. In addition to a physically settled dayahead spot market, Nord Pool provides a financially settled future market. Unlike in other markets, futures on electricity at Nord Pool trade only up to the start of a so called delivery

\footnotetext{
${ }^{*}$ Fred Espen Benth, Centre of Mathematics for Applications, University of Oslo, P.O. Box 1053, Blindern, N-0316 Oslo, Norway. Email: fredb@math.uio.no

${ }^{\dagger}$ Nils Detering, Department of Mathematics, University of Munich, Theresienstraße 39, 80333 Munich. Germany. Email: n.detering@math.lmu.de

${ }^{\ddagger}$ Nils Detering would like to thank the Centre of Mathematics for Applications(CMA) in Oslo for the hospitality during the time when most of this work was done. Fred Espen Benth acknowledges financial support from the project "Managing Weather Risk in Electricity Markets (MAWREM)" funded by the RENERGI program of the Norwegian Research Council. We would like to thank Natalie Packham for helpful suggestions. Two anonymous referees and the associate editor are thanked.
} 
period, imposing restrictions on the possible trading strategies when using these instruments for hedging options.

Denote by $\left(S_{t}\right)_{0 \leq t \leq T_{2}}$ the spot energy price. Traded future contracts in energy markets allow to fix the average price of energy over a certain time period $\left[T_{1}, T_{2}\right]$, the delivery period, by defining a payout

$$
\frac{1}{T_{2}-T_{1}} \int_{T_{1}}^{T_{2}} S_{r} \mathrm{~d} r
$$

in return for the agreed future price 11 This is in contrast to other markets where a future allows to fix the price at a single time point. The reason for using the average price lies in the nature of production and consumption of energy that naturally happens over a time period. Additionally one has usually $0<T_{1}$, meaning the delivery period starts in the future, to account for seasonality in energy prices. Nord Pool offers trading in futures with different length of delivery periods (week, month, quarter, year) and different starting dates. The future contract with payout described in Equation (1.1) is only traded up to $T_{1}$. Within the delivery period $\left(T_{1}, T_{2}\right]$ trading on the exchange is not possible. The same trading restriction applies to several natural gas futures traded at the Chicago Mercantile Exchange (CME), where our results can also be applied. Energy futures at CME may be traded in the delivery period but the trading volume is restricted to whole number multiples of reference days in the delivery period. In other markets, for example at the European Energy Exchange (EEX) in Germany, trading is allowed during the delivery period without restriction but liquidity is very low.

The trading restriction becomes important when using the future as a hedging instrument for options on energy spot.

The aim of this paper is the hedging of options settled against the average spot, i.e. $\mathcal{F}_{T_{2}}$ measurable payoffs $H$ of the form

$$
H=h\left(\frac{1}{T_{2}-T_{1}} \int_{T_{1}}^{T_{2}} S_{r} \mathrm{~d} r\right)
$$

with some Borel measurable function $h$. Most commonly traded are call and put payoffs. These options are generally known as Asian-style options.

The available literature on Asian options is mainly focused on financial markets, as equity or fixed income, where the process $S_{t}$ subject to averaging is a tradable asset. In (Vecer, 2001) and (Vecer and $\mathrm{Xu}, 2004$ ) it is shown that the avarage spot price can be replicated by dynamically trading in $S_{t}$. That way a pricing PDE for options on the average spot price can be derived. This however crucially relies on the fact that the underlying spot is a tradable asset. In energy markets the spot is a typical non-tradable asset, since energy cannot be stored. Additionally, the situation in energy markets is special since futures with delivery period coinciding with the averaging period of the options are available for trading. Therefore, an option on the average spot can essentially be considered as an option on the final value of the future and as such, can be hedged by dynamically trading this future. Due to the trading restriction in the delivery period as mentioned above, it is in general not possible to perfectly hedge the claim and one can only aim at finding risk minimizing strategies. The only work on Asian options in energy is (Weron, 2008) to our knowledge. In the paper, a pricing measure is found by jointly analyzing traded future and spot prices. This measure is then used to calculate prices for Asian options. The prices are compared with the prices of traded Asian options at Nord Pool and show a good fit. However, hedging, and the impact of the trading restriction is not considered there.

In this paper we explicitly solve the problem of pricing and hedging, taking this trading restriction into account. We work in the spirit of (Föllmer and Schweizer, 1991), (Schweizer,

\footnotetext{
${ }^{1}$ In actual trading at Nord Pool the average price is based on hourly prices and becomes a discrete sum.
} 
1994) and (Schweizer, 1995b) in using a quadratic risk criterion to minimize the resulting hedge error. We show for a rather general type of processes that a unique solution exists to this combined continuous-discrete hedging problem. Additionally we show that the hedge positions for $t<T_{1}$ are not affected by the trading restrictions if the process is a local martingale.

Based on our general results, we explicitly derive the hedge positions for processes often used to model energy future prices. As a simple example we consider a Black-Scholes model with time dependent volatility. Such a model is still frequently used in the market and offers closed form solutions for put and call payoffs. As models that resemble a more realistic picture of the price process in energy we consider a version of the Barndorff-Nielsen and Shepard model and an exponential additive model. For these models we derive integral representations of the hedge positions. The hedge positions for $t<T_{1}$ in the exponential additive model are derived from recent results in (Goutte et al., 2014). Finally we model the spot price by an extension of the Schwarz-Smith model (Schwartz and Smith, 2000), that allows the driving long and short term factors to be Lévy processes instead of Brownian motions. Based on this spot dynamics we show how one can derive an exponential additive model for the future price by moment matching if the two Lévy processes are Normal Inverse Gaussian (NIG) distributed. To state a model for the future is not straightforward due to the missing market prices in $\left(T_{1}, T_{2}\right]$ and a model implied by the spot prices, which can be observed at any time, appears reasonable. Finally we estimate our model based on historical spot and future prices and analyze the distribution of the hedge error in a simulation study.

While we focus in the paper on Asian-style options on energy our results can also be applied to the hedging of so called quanto option $2^{2}$. Quanto options have recently become very popular and allow a hedge against two risk factors, price risk and volume risk, that is, the risk that both, demand and prices for energy changes. For example in a very warm winter, there will be less demand for heating energy than expected, which in turn will also lower prices. The volume level is usually approximated by the number of days with a temperature above or below a certain threshold (see (Benth et al., 2014+) for a detailed account on Quanto options).

The paper is organized as follows. In Section 2 we show that there exists a unique solution to the pricing and hedging problem in a special semimartingale setting. Additionally we show that the hedge positions for $t<T_{1}$ remain unchanged despite the trading restriction after $T_{1}$ if the future process is a local martingale. In Section 3 we look at three models that are frequently used in energy and derive the hedge positions in these models, a Black-Scholes model with timedependent but deterministic volatility, a version of the Barndorff-Nielsen and Shepard model and an exponentially additive model. Section 4 comments on the accrual of interest rates on margin accounts. In Section 5 we specify a model for the future price based on moment matching and estimate its parameters. We provide a simulation study to demonstrate the magnitude of the hedge error incurred by the trading restriction analyzed in our paper.

\section{Theoretical setup and existence of solution}

We work on a probability space $\left(\Omega, \mathbb{P},\left(\mathcal{F}_{t}\right)_{0 \leq t \leq T_{2}}\right)$ on which an adapted process $\left(X_{t}\right)_{0 \leq t \leq T_{2}}$ on $\mathbb{R}$ is defined that is a $\mathbb{P}$-semimartingale and represents the price process of our primary security used for hedging, in our case a future contract that can be entered at zero cost. We shall choose a càdlàg version of $X$ and further assume that $X$ is special with decomposition

$$
X=X_{0}+M+\int \alpha \mathrm{d}[M, M]
$$

\footnotetext{
${ }^{2}$ The term quanto option is also used in other markets to denote options that have an additional cross currency option included. However, the quanto options traded in energy are of rather different nature.
} 
where $M$ is a square integrable local martingale and $\alpha$ predictable. This especially implies that the predictable finite variation component of $X$ is absolutely continuous with respect to the quadratic variation of the martingale component. Additionally, we assume that the meanvariance tradeoff process of $X$ defined by

$$
K_{t}:=\int_{0}^{t} \alpha_{s}^{2} \mathrm{~d}[M, M]_{s}
$$

is bounded. If $K_{t}$ is only assumed to be finite $\mathbb{P}$-almost surely, this is the so-called Structure Condition and related to no-arbitrage as shown in (Schweizer, 1992). Additionally we are given an $\mathcal{F}_{T_{2}}$ measurable payoff $H$ with the property $\mathbb{E}\left[H^{2}\right]<\infty$. We define for the rest of the paper the payoff function of a European call and put option by

$$
\begin{aligned}
& C\left(X_{T_{2}}\right):=\left(X_{T_{2}}-K\right)^{+} \\
& P\left(X_{T_{2}}\right):=\left(X_{T_{2}}-K\right)^{-} .
\end{aligned}
$$

Remember that $X_{T_{2}}$ is the final value of the future with payout (1.1), such that the payoffs $C\left(X_{T_{2}}\right)$ and $P\left(X_{T_{2}}\right)$ are essentially Asian-style options on the spot price of the type 1.2 .

We assume that trading is continuously possible up to $T_{1}<T_{2}$. The trading position in $\left[T_{1}, T_{2}\right]$ has to be constant. This trading restriction introduces a market incompleteness, even if the model would be complete without the restriction.

The hedge portfolio $\left(V_{t}\right)_{0 \leq t \leq T_{2}}$ is then given by

$$
V_{t}=V_{0}+\int_{0}^{T_{1} \wedge t} \psi_{s} \mathrm{~d} X_{s}+\mathbf{1}_{\left\{t>T_{1}\right\}} \psi_{T_{1}}\left(X_{t}-X_{T_{1}}\right)
$$

where $\psi_{s}$ is predictable and fulfills the following integrability condition (IC):

$$
\mathbb{E}\left[\int_{0}^{T_{1}} \psi_{s}^{2} \mathrm{~d}[M, M]_{s}+\psi_{T_{1}}^{2}\left([M, M]_{T_{2}}-[M, M]_{T_{1}}\right)\right]<\infty .
$$

The property (2.6) has the effect that the trading gains and therefore the portfolio process $V_{t}$ is also a special semimartingale and that there is no arbitrage. Usually one would require additionally that $\psi$ is such that $\int|\psi \alpha| \mathrm{d}[M, M]$ is integrable. However, as shown in (Schweizer, 1994), this assumption is redundant if $K_{t}$ is bounded. Note that since trading is done in a future contract that can be entered at zero cost, no further self-financing constraints are needed 3

We aim to find a pair $\left(V_{0},\left(\psi_{t}\right)_{0 \leq t \leq T_{1}}\right)$ that minimizes the expected quadratic hedge error

$$
\mathbb{E}\left[\left(H-V_{T_{2}}\right)^{2}\right] .
$$

This problem has been extensively studied for the situation without trading restriction where $T_{1}=T_{2}$. The theoretical problem was solved in the seminal papers (Föllmer and Sondermann, 1986) for the martingale case and in (Föllmer and Schweizer, 1991), (Schweizer, 1995a) and (Schweizer, 1994) for general semimartingales. The theory for quadratic hedging when the underlying process is discrete was developed in (Schäl, 1994) and in more generality in (Schweizer, 1995b). An algorithm for explicit computation of hedge positions in discrete time is proposed and applied to energy markets in (Goutte et al., 2014+). However, our situation is a mixture of both and not captured by the current literature.

\footnotetext{
${ }^{3}$ To avoid double counting of jumps at time $T_{1}$, one could argue that the integral should only go up to $T_{1}-$. However, since there are no financial market models with fixed times of jumps, we can choose $T_{1}$ instead for convenience without changing $V_{T_{2}}$ under $\mathbb{P}$.
} 
First we show existence of a unique solution to the minimization problem (2.7). For doing this we follow a similar path as in the situation without trading restrictions by projection techniques on a subspace of the Hilbert space $\mathcal{L}^{2}(\mathbb{P})$ of square integrable $\mathcal{F}_{T_{2}}$ measurable random variables. Therefore we equip $\mathcal{L}^{2}(\mathbb{P})$ with the usual inner product

$$
\left(N_{1}, N_{2}\right):=\mathbb{E}\left[N_{1} N_{2}\right] \text { for } N_{1}, N_{2} \in \mathcal{L}^{2}(\mathbb{P})
$$

and the resulting norm

$$
\left\|N_{1}\right\|=\mathbb{E}\left[N_{1}^{2}\right]^{1 / 2}<\infty .
$$

Under this scalar product $\mathcal{L}^{2}(\mathbb{P})$ becomes a Hilbert space. We aim to find a representation 2.5 by projecting the square integrable payoff $H$ on the subspace of $\mathcal{L}^{2}(\mathbb{P})$ that is generated by trading strategies of the form 2.5 . We thus have to show that the set $\{G(\psi)\}$ of $\mathcal{F}_{T_{2}}$ measurable random variables with $G(\psi)$ of the form

$$
G(\psi)=\int_{0}^{T_{1}} \psi_{s} \mathrm{~d} X_{s}+\psi_{T_{1}}\left(X_{T_{2}}-X_{T_{1}}\right)
$$

and $\psi$ predictable, fulfilling $(\mathbf{I C})$ is a closed linear subspace of the Hilbert space $\mathcal{L}^{2}(\mathbb{P})$. This is done in Lemma 2.2 below for integration with respect to $M$, the local martingale component of $X$ and using Lemma 2.3 extended to $X$. Before proving the closure property we shall prove the following lemma about integration with respect to local martingales, which is usually only stated for martingales but extends easily.

Lemma 2.1. Let $M$ be a local martingale and $\phi$ predictable such that $\mathbb{E}\left[\int_{0}^{T} \phi_{s}^{2} \mathrm{~d}[M, M]_{s}\right]<\infty$, then $N_{t}:=\int_{0}^{T \wedge t} \phi_{s} \mathrm{~d} M_{s}$ is a square integrable martingale and $\mathbb{E}\left[\left(\int_{0}^{T} \phi_{s} \mathrm{~d} M_{s}\right)^{2}\right]=\mathbb{E}\left[\int_{0}^{T} \phi_{s}^{2} \mathrm{~d}[M, M]_{s}\right]$.

Proof. First observe that the process defined by $N_{t}:=\int_{0}^{T \wedge t} \phi_{s} \mathrm{~d} M_{s}$ is a local martingale. To see this, let $\bar{T}_{n}$ be a stopping sequence reducing $M$. Then $\bar{T}_{n} \wedge T$ reduces $M$ and one can see that it also reduces $N$ by preservation of the martingale property when integrating with respect to $M_{t \wedge\left(\bar{T}_{n} \wedge T\right)}$. Because $N$ stays constant after $T$ we find that also $\bar{T}_{n}$ reduces $N$ and $N$ is therefore a local martingale. Additionally by properties of the quadratic variation we know that $[\phi \cdot M, \phi \cdot M]_{t}=\int_{0}^{t} \phi_{s}^{2} \mathrm{~d}[M, M]$, which implies that $\mathbb{E}\left[[\phi \cdot M, \phi \cdot M]_{t}\right]<\infty$ for all $t \leq T$ by assumption on $\phi$. However, this implies that $N$ is actually a square integrable martingale (Protter, 2005, Corollary 3, Chapter 2.6) and the Itô isometry follows from partial integration.

For the rest of this section we introduce some notation. For a set of predictable processes $\theta$ and a semimartingale $S$ denote by $\mathcal{N}_{\theta, S}$ the set of random variables generated by integrating elements in $\theta$ with respect to $S$, i.e. $\mathcal{N}_{\theta, S}:=\left\{N: N=\int_{0}^{T_{2}} \psi_{r} \mathrm{~d} S_{r}\right.$ for some $\left.\psi \in \theta\right\}$. In the following the semimartingale will be either $X$ or the local martingale component $M$ of $X$ and the set $\theta$ will always be such that $\mathcal{N}_{\theta, S} \subset \mathcal{L}^{2}(\mathbb{P})$. Let further $\theta_{1}$ and $\theta_{2}$ be given by

$$
\begin{aligned}
& \theta_{1}:=\left\{\psi \text { predictable }: \psi_{s}=0 \text { for } s \geq T_{1}, \mathbb{E}\left[\int_{0}^{T_{1}} \psi_{s}^{2} \mathrm{~d}[M, M]_{s}\right]<\infty\right\} \\
& \theta_{2}:=\left\{\psi \text { predictable }: \psi_{s}=\mathbf{1}_{\left\{s \geq T_{1}\right\}} \zeta, \mathbb{E}\left[\zeta^{2}\left([M, M]_{T_{2}}-[M, M]_{T_{1}}\right)\right]<\infty\right\} .
\end{aligned}
$$

Note that since $\psi \in \theta_{2}$ is supposed to be predictable this implies that the element $\zeta$ defining $\psi$ is $\mathcal{F}_{T_{1}-}$ measurable. The set $\theta_{1}$ consists of possible trading strategies up to $T_{1}$ and $\theta_{2}$ of discrete trading strategies after $T_{1}$. The next two lemmas show that the subspace of $\mathcal{L}^{2}(\mathbb{P})$ generated by the set of possible trading strategies $\theta_{1}+\theta_{2}$ is closed. 
Lemma 2.2. Let $M$ be the square integrable local martingale component of $X$. Then $\mathcal{N}_{\theta_{1}+\theta_{2}, M}$ is a closed linear subspace of $\mathcal{L}^{2}(\mathbb{P})$. Further, the two subspaces $\mathcal{N}_{\theta_{1}, M}$ and $\mathcal{N}_{\theta_{2}, M}$ are orthogonal and $\mathcal{N}_{\theta_{1}+\theta_{2}, M}=\mathcal{N}_{\theta_{1}, M} \oplus \mathcal{N}_{\theta_{2}, M}$.

Proof. It clearly is a subspace and $\mathcal{N}_{\theta_{1}+\theta_{2}, M}=\mathcal{N}_{\theta_{1}, M}+\mathcal{N}_{\theta_{2}, M}$. To show that it is closed we show that the two subspaces $\mathcal{N}_{\theta_{1}, M}$ and $\mathcal{N}_{\theta_{2}, M}$ are closed and orthogonal, which implies that also the sum is closed and that it is direct.

1. The space $\mathcal{N}_{\theta_{1}, M}$ is closed because the integration map is a Hilbert space isomorphism between the two Hilbert spaces $\theta_{1}$ with the scalar product $(\psi, \phi):=\mathbb{E}\left[\int_{0}^{T_{1}} \psi_{s} \phi_{s} \mathrm{~d}[M, M]\right.$ and the space $\mathcal{N}_{\theta_{1}, M}$ together with the scalar product defined in 2.8 by the Lemma 2.1

2. We have to show that $\mathcal{N}_{\theta_{2}, M}$ is also a closed subspace with respect to the scalar product defined in (2.8). We adapt the arguments in (Schweizer, 1995b, Theorem 2.1.). Let $\left\{N_{n}\right\}$ be a Cauchy sequence in $\mathcal{N}_{\theta_{2}, M}$, then there exists a corresponding sequence $\left\{\psi_{n}\right\}$ with $N_{n}=\psi_{n}\left(M_{T_{2}}-M_{T_{1}}\right)$ and $\mathbb{E}\left[\psi_{n}^{2}\left([M, M]_{T_{2}}-[M, M]_{T_{1}}\right)\right]<\infty$. Since $N_{n}$ is a Cauchy sequence, we find $\left\|\psi_{n}\left(M_{T_{2}}-M_{T_{1}}\right)-\psi_{m}\left(M_{T_{2}}-M_{T_{1}}\right)\right\|^{2}=\mathbb{E}\left[\left(\psi_{n}-\psi_{m}\right)^{2}\left(M_{T_{2}}-M_{T_{1}}\right)^{2}\right] \leq \epsilon$ for $m, n>M_{\epsilon}$. But since

$$
\mathbb{E}\left[\left(\psi_{n}-\psi_{m}\right)^{2}\left(M_{T_{2}}-M_{T_{1}}\right)^{2}\right]=\mathbb{E}\left[\left(\psi_{n}-\psi_{m}\right)^{2} \mathbb{E}\left[\left(M_{T_{2}}-M_{T_{1}}\right)^{2} \mid \mathcal{F}_{T_{1}-}\right]\right]
$$

by the tower law and the fact that $\left(\psi_{n}-\psi_{m}\right)^{2}$ is $\mathcal{F}_{T_{1}}$ - measurable, it follows that also $\psi_{i} \sqrt{\mathbb{E}\left[\left(M_{T_{2}}-M_{T_{1}}\right)^{2} \mid \mathcal{F}_{T_{1}-}\right]}$ is Cauchy, converging to some square integrable $\mathcal{F}_{T_{1}}$ measurable $\zeta_{\infty}$. Choosing

$$
\psi_{\infty}:=\mathbf{1}_{\left\{\mathbb{E}\left[\left(M_{T_{2}}-M_{T_{1}}\right)^{2} \mid \mathcal{F}_{T_{1}-}\right]>0\right\}} \frac{\zeta_{\infty}}{\sqrt{\mathbb{E}\left[\left(M_{T_{2}}-M_{T_{1}}\right)^{2} \mid \mathcal{F}_{T_{1}-}\right]}}
$$

then $\psi_{\infty}\left(M_{T_{2}}-M_{T_{1}}\right) \in \mathcal{L}^{2}(\mathbb{P})$ and

$$
\begin{aligned}
\mathbb{E}\left[\left(\psi_{n}-\psi_{\infty}\right)^{2}\left(M_{T_{2}}-M_{T_{1}}\right)^{2}\right] & =\mathbb{E}\left[\left(\psi_{n}-\psi_{\infty}\right)^{2} \mathbb{E}\left[\left(M_{T_{2}}-M_{T_{1}}\right)^{2} \mid \mathcal{F}_{T_{1}-}\right]\right] \\
& =\left\|\psi_{n} \sqrt{\mathbb{E}\left[\left(M_{T_{2}}-M_{T_{1}}\right)^{2} \mid \mathcal{F}_{T_{1}}\right]}-\zeta_{\infty}\right\|
\end{aligned}
$$

such that $N_{n}$ converges to $\psi_{\infty}\left(M_{T_{2}}-M_{T_{1}}\right)$ in $\mathcal{L}^{2}(\mathbb{P})$. By Lemma 2.1 and the same argument as in 1 ., we find that $\mathbb{E}\left[\psi_{\infty}\left([M, M]_{T_{2}}-[M, M]_{T_{1}}\right)\right]<\infty$ showing that $\mathcal{N}_{\theta_{2}, M}$ is closed. The indicator function in defining $\psi_{\infty}$ is needed to have the expression well defined if $\mathbb{E}\left[\left(M_{T_{2}}-M_{T_{1}}\right)^{2} \mid \mathcal{F}_{T_{1}-}\right]=0$, which is for example the case if $M_{t}$ is constant for $t \in\left[T_{1}, T_{2}\right]$.

3. Now, we show orthogonality of the two spaces, from which it follows that the direct sum is closed. For orthogonality, we calculate for $\int_{0}^{T_{1}} \psi_{s} \mathrm{~d} M_{s} \in \mathcal{N}_{\theta_{1}, M}$ and $\psi_{T_{1}}\left(M_{T_{2}}-M_{T_{1}}\right) \in$ $\mathcal{N}_{\theta_{2}, M}$ using that $\int_{0}^{T_{1}} \psi_{s} \mathrm{~d} M_{s}$ and $\psi_{T_{1}}$ is $\mathcal{F}_{T_{1}}$-measurable

$$
\begin{aligned}
\left(\int_{0}^{T_{1}} \psi_{s} \mathrm{~d} M_{s}, \psi_{T_{1}}\left(M_{T_{2}}-M_{T_{1}}\right)\right) & =\mathbb{E}\left[\left(\int_{0}^{T_{1}} \psi_{s} \mathrm{~d} M_{s}\right) \psi_{T_{1}}\left(M_{T_{2}}-M_{T_{1}}\right)\right] \\
& =\mathbb{E}\left[\mathbb{E}\left[\left(\int_{0}^{T_{1}} \psi_{s} \mathrm{~d} M_{s}\right) \psi_{T_{1}}\left(M_{T_{2}}-M_{T_{1}}\right) \mid \mathcal{F}_{T_{1}}\right]\right] \\
& =\mathbb{E}\left[\left(\int_{0}^{T_{1}} \psi_{s} \mathrm{~d} M_{s}\right) \mathbb{E}\left[\psi_{T_{1}}\left(M_{T_{2}}-M_{T_{1}}\right) \mid \mathcal{F}_{T_{1}}\right]\right] \\
& =0
\end{aligned}
$$


since $\mathbb{E}\left[\psi_{T_{1}}\left(M_{T_{2}}-M_{T_{1}}\right) \mid \mathcal{F}_{T_{1}}\right]=0$ by Lemma 2.1. Therefore the both subspaces are orthogonal.

4. From the orthogonality it follows that $\mathcal{N}=\mathcal{N}_{\theta_{1}, M} \oplus \mathcal{N}_{\theta_{2}, M}$ and additionally, together with the fact that both spaces are closed, that $\mathcal{N}_{\theta_{1}+\theta_{2}, M}$ is closed ${ }^{4}$

The following lemma shows that the closure property extends to integration with respect to $X$.

Lemma 2.3. Define

$$
\Theta:=\left\{\phi \text { predictable }: \mathbb{E}\left[\int_{0}^{T_{2}} \phi_{s}^{2} \mathrm{~d}[M, M]_{s}\right]<\infty\right\},
$$

where $M$ is the local martingale component of $X$. Further, let $\theta$ be a subset $\theta \subset \Theta$. Then $\mathcal{N}_{\theta, X}$ is a closed subspace of $\mathcal{L}^{2}(\mathbb{P})$ if and only if $\mathcal{N}_{\theta, M}$ is a closed subspace of $\mathcal{L}^{2}(\mathbb{P})$.

Proof. It is shown in (Monat and Stricker, 1994) that the two norms on $\Theta$ defined by

$$
\|\phi\|_{L(X)}:=\left\|\int_{0}^{T} \phi_{s} \mathrm{~d} X_{s}\right\| \text { and }\|\phi\|_{L(M)}:=\left\|\int_{0}^{T} \phi_{s} \mathrm{~d} M_{s}\right\|
$$

are equivalent. Therefore both $\mathcal{N}_{\theta, X}$ and $\mathcal{N}_{\theta, M}$ are subsets of $\mathcal{L}^{2}(\mathbb{P})$ and clearly, $\mathcal{N}_{\theta, X}$ is a subspace of $\mathcal{L}^{2}(\mathbb{P})$ if and only if $\mathcal{N}_{\theta, M}$ is a subspace of $\mathcal{L}^{2}(\mathbb{P})$. Assume $\mathcal{N}_{\theta, M}$ is closed and let $N^{i}$ be a Cauchy sequence in $\mathcal{N}_{\theta, M}$, then there exists a corresponding integrand $\phi^{i}$ such that $N^{i}=\int_{0}^{T} \phi^{i} \mathrm{~d} X$. By the norm equivalence it follows that also $M^{i}:=\int_{0}^{T} \phi^{i} \mathrm{~d} X$ is a Cauchy sequence. Since $\mathcal{N}_{\theta, M}$ is known to be closed, the sequence $M^{i}$ has a limit $M^{\infty}$ of the form $M^{\infty}:=\int_{0}^{T} \phi^{\infty} \mathrm{d} M$. Define $N^{\infty}:=\int_{0}^{T} \phi^{\infty} \mathrm{d} X \in \mathcal{N}_{\theta, M}$. Again by norm equivalence it follows that $N^{i}$ converges to $N^{\infty}$ as $i$ goes to infinity. The only if part can be shown by reverting the argument.

With the help of the last two lemmas we obtain directly the existence of a unique solution to 2.7) in terms of the final portfolio value $V_{T_{2}}$ as summarized in the next proposition. Let in the following $\Pi^{Y}$ be the projection operator on a closed subspace $Y \subset \mathcal{L}^{2}(\mathbb{P})$.

Proposition 2.4. For each $V_{0} \in \mathbb{R}$ and $H$ with $\mathbb{E}\left[H^{2}\right]<\infty$ there exists a unique solution $G(\psi) \in \mathcal{N}_{\theta_{1}+\theta_{2}, X}$ that minimizes

$$
\inf _{G(\phi) \in \mathcal{N}_{\theta_{1}+\theta_{2}, X}} \mathbb{E}\left[\left(\left(H-V_{0}\right)-G(\phi)\right)^{2}\right] .
$$

If $X$ is a square integrable local martingale, that is $\alpha=0$ then $G(\psi)$ does not depend on $V_{0}$ and the optimal initial capital $V_{0}$ is given by $\mathbb{E}[H]$.

Proof. We know from Lemma 2.2 that $\mathcal{N}_{\theta_{1}+\theta_{2}, M}$ is closed. This extends by Lemma 2.3 to $\mathcal{N}_{\theta_{1}+\theta_{2}, X}$, so $\mathcal{N}_{\theta_{1}+\theta_{2}, X}$ is a closed subspace of $\mathcal{L}^{2}(\mathbb{P})$. Then, since $H-V_{0} \in \mathcal{L}^{2}(\mathbb{P})$ there exists a unique $G(\psi) \in N_{\theta_{1}+\theta_{2}, X}$ such that

$$
\inf _{N \in \mathcal{N}_{\theta_{1}+\theta_{2}, X}}\left\|\left(H-V_{0}\right)-N\right\|=\left\|\left(H-V_{0}\right)-G(\psi)\right\|
$$

\footnotetext{
${ }^{4}$ We use $N=N_{1} \oplus N_{2}$ to denote the direct sum between two subspaces $N_{1}$ and $N_{2}$, that is $N_{1} \cap N_{2}=\emptyset$ and $N=\left\{x+y \mid x \in N_{1}, y \in N_{2}\right\}$. Sometimes the same symbol is used when the two subspaces are additionally assumed to be orthogonal.
} 
and $H-V_{0}=G(\psi)+L^{H+V_{0}}$ with $G(\psi)$ given by the orthogonal projection $G(\psi):=\Pi^{\mathcal{N}_{\theta_{1}+\theta_{2}, X}}(H-$ $V_{0}$ ) and $L^{H-V_{0}} \perp G(\psi)$ (see (Yosida, 1980, Theorem 1, Chapter 3)). If $X$ is a square integrable local martingale, then $\Pi^{\mathcal{N}_{\theta_{1}+\theta_{2}, X}}\left(V_{0}\right)=\Pi^{\mathcal{N}_{\theta_{1}+\theta_{2}, M}}\left(V_{0}\right)=0$ since $\left(V_{0}, G(\psi)\right)=\mathbb{E}\left[V_{0} G(\psi)\right]=$ $V_{0} \mathbb{E}[G(\psi)]=0, \forall G(\psi) \in N_{\theta_{1}+\theta_{2}, M}$ by Lemma 2.1 such that the strategy does not depend on the initial capital. The remaining risk $L^{H-V_{0}}$ however does, since due to the orthogonality

$$
\left\|L^{H-V_{0}}\right\|^{2}=\left\|H-V_{0}\right\|^{2}-\|G(\psi)\|^{2} .
$$

Since $\|G(\psi)\|^{2}$ does not depend on $V_{0},\left\|L^{H-V_{0}}\right\|^{2}$ is minimized if $\left\|H-V_{0}\right\|^{2}=\mathbb{E}\left[\left(H-V_{0}\right)^{2}\right]$ is minimized. But $V_{0}=\mathbb{E}[Y]$ minimizes $\left\{\mathbb{E}\left[\left(Y-V_{0}\right)\left(Y-V_{0}\right)\right] \mid V_{0} \in \mathbb{R}\right\}$ for any square integrable random variable $Y$, such that the risk is minimized by an initial capital of $V_{0}=\mathbb{E}[H]$.

So far we have shown uniqueness in terms of the square integrable variable $G(\psi)$ minimizing (2.14), but we did not analyze if $\psi$ is actually unique. The next proposition provides uniqueness for $\psi$.

Proposition 2.5. The process $\left(\psi_{t}\right)_{0 \geq t \geq T_{2}}$ is unique with respect to the measure $\mu_{[M, M]}$ defined on $\left[0, T_{2}\right] \times \Omega$ by

$$
\mu_{[M, M]}((s, t] \times B)=\mathbb{E}\left(\mathbf{1}_{B}\left([M, M]_{t}-[M, M]_{s}\right) .\right.
$$

Proof. Assume that $\psi \neq \tilde{\psi}$, but $G(\psi)=G(\tilde{\psi})$. Then

$$
\int_{0}^{T_{2}}\left(\psi_{s}-\tilde{\psi}_{s}\right) \mathrm{d} X_{s}=0
$$

and it follows that

$$
\mathbb{E}\left[\left(\int_{0}^{t}\left(\psi_{s}-\tilde{\psi}_{s}\right) \mathrm{d} X_{s}\right)^{2}\right]=0 .
$$

Again using the norm equivalence (Monat and Stricker, 1994) we get that also

$$
\mathbb{E}\left[\left(\int_{0}^{t}\left(\psi_{s}-\tilde{\psi}_{s}\right) \mathrm{d} M_{s}\right)^{2}\right]=\mathbb{E}\left[\int_{0}^{t}\left(\psi_{s}-\tilde{\psi}_{s}\right)^{2} \mathrm{~d}[M, M]_{s}\right]=0
$$

where $M$ is the local martingale part of $X$ and where the first equality follows again by Itô isometry. The process $[M, M]$ is of finite variation on compacts, which implies that $\int_{0}^{t}\left(\psi_{s}-\right.$ $\left.\tilde{\psi}_{s}\right)^{2} \mathrm{~d}[M, M]_{s}$ is equal to the Lebesgue-Stiltjes integral. Since $[M, M]$ is non-decreasing and $\left(\psi_{s}-\tilde{\psi}_{s}\right)^{2}$ positive this implies that $\int_{0}^{t}\left(\psi_{s}-\tilde{\psi}_{s}\right)^{2} \mathrm{~d}[M, M]_{s}=0$, $\mathbb{P}$-a.s.. It follows that $\psi-\tilde{\psi}=0$ with respect to the measure defined in 2.15.

Especially if the measure generated by $[M, M]$ is absolutely continuous with respect to the Lebesgue measure, then $\psi$ is unique for a.a. $(t, \omega) \in\left[0, T_{2}\right] \times \Omega$.

In the following we shall restrict to the situation where $\alpha=0$, that is $X$ is a local martingale $\left(X=X_{0}+M\right)$. There are two reasons to consider this simplified model, which in turn allows for stronger results. First, there is empirical evidence that the future price process in energy has very small drift, even under the objective measure. For example (Benth et al., 2008, Table 8.3) find that the mean return on the future is essentially zero. Second, especially in incomplete markets, available options are not redundant. They can be used for static hedges to considerably reduce hedging risk. When options are partly statically hedged with benchmark options, the cost of replication has to be incorporated into the pricing rule. For that reason, it is industry standard even in an incomplete market to chose an equivalent martingale measure to calculate prices and hedge ratios. This equivalent martingale measure is chosen in a trade-off between 
market calibration and resemblance of the statistical behavior of the asset process (see (Cont and Tankov, 2004, Chapter 10.6 and Chapter 13) for discussions). Therefore from a practical perspective hedging under a martingale measure is the most relevant. To avoid confusion, in the following we shall use $\mathbb{Q}$ to denote the measure that describes the future price process, but the reader should be aware that, despite being a martingale measure, it could be the objective probability measure.

An immediate question one might ask is whether the value of the hedge portfolio for $t<T_{1}$ is actually the same as the one resulting from the quadratic risk minimizing problem without the trading restriction in $\left(T_{1}, T_{2}\right]$. The following proposition shows that this is in fact the case.

Proposition 2.6. Let $H$ be an $\mathcal{F}_{T_{2}}$ measurable claim with $\mathbb{E}\left[H^{2}\right]<\infty$ and let $G(\psi)_{0 \leq t \leq T_{2}}$ be the trading gains from the optimal hedging strategy without trading restrictions, which in the martingale case is given by $G(\psi)_{t}=\mathbb{E}\left[G(\phi) \mid \mathcal{F}_{t}\right]$. Then $G(\psi)_{0 \leq t<T_{1}}$ are the trading gains from the optimal strategy when trading is restricted for $t \in\left(T_{1}, T_{2}\right]$.

Proof. Let $\Theta$ be defined as in Lemma 2.3. By the same arguments as in Lemma 2.2 for $\mathcal{N}_{\theta_{1}, M}$ the space $\mathcal{N}_{\Theta, M}$ is a closed linear subspace of $\mathcal{L}^{2}(\mathbb{P})$. We have $\mathcal{N}_{\theta_{1}, M} \subset \mathcal{N}_{\Theta, M}$. To see this let $N \in \mathcal{N}_{\theta_{1}, M}$ and let $(\phi)_{0 \leq t \leq T_{1}}$ be an integrand that generates $N$. Then $\bar{\phi}:=\phi_{\mathbf{1}_{t \leq T_{1}}}+0_{\mathbf{1}_{t>T_{1}}}$ is a valid integrand for $\mathcal{N}_{\Theta, M}$ since $\mathbb{E}\left[\int_{0}^{T_{2}} \bar{\phi}_{s}^{2} \mathrm{~d}[M, M]\right]=\mathbb{E}\left[\int_{0}^{T_{1}} \phi_{s}^{2} \mathrm{~d}[M, M]\right]<\infty$ and clearly $N=\int_{0}^{T_{2}} \bar{\phi}_{s} \mathrm{~d} M_{s}$, thus $\mathcal{N}_{\theta_{1}, M} \subset \mathcal{N}_{\Theta, M}$. Since $\mathcal{N}_{\theta_{1}, M}$ is a subspace and closed, we obtain that $\mathcal{N}_{\Theta, M}=\mathcal{N}_{\theta_{1}, M} \oplus \mathcal{N}_{\theta_{1}, M}^{\perp}$. Additionally we have $\mathcal{N}_{\theta_{2}, M} \subset \mathcal{N}_{\theta_{1}, M}^{\perp}$ and therefore $\mathcal{N}_{\theta_{1}+\theta_{2}, M}=$ $\mathcal{N}_{\theta_{1}, M} \oplus \mathcal{N}_{\theta_{2}, M} \subset \mathcal{N}_{\theta_{1}, M} \oplus \mathcal{N}_{\theta_{1}, M}^{\perp}$. Then $G(\psi)=\Pi^{\mathcal{N}} \mathcal{N}_{\Theta, M}\left(H-V_{0}\right) \in \mathcal{N}_{\Theta, M}$ represents the final value of the optimal strategy without trading restrictions. It can be decomposed as $G(\psi)=N_{1}+N_{2}$ with $N_{1} \in \mathcal{N}_{\theta_{1}, M}, N_{2} \in \mathcal{N}_{\theta_{1}, M}^{\perp}$. Since $\mathbb{E}\left[N_{2} \mid \mathcal{F}_{t}\right]=0$ for $t \leq T_{1}$, it follows

$$
G(\psi)_{t}=\mathbb{E}\left[N_{1} \mid \mathcal{F}_{t}\right], \text { for } t \leq T_{1} .
$$

Since $\Pi^{X}\left(\Pi^{Y}(x)\right)=\Pi^{X}(x)$ for subspaces $X \subset Y$, it follows that $\Pi^{\mathcal{N}_{\theta_{1}, M} \oplus \mathcal{N}_{\theta_{2}, M}}\left(N_{1}+N_{2}\right)$ provides the final value of the trading strategy in the restricted case and calculates as $\tilde{G}(\tilde{\psi}):=$ $\Pi^{\mathcal{N}_{\theta_{1}, M} \oplus \mathcal{N}_{\theta_{2}, M}}\left(N_{1}+N_{2}\right)=\Pi^{\mathcal{N}_{\theta_{1}, M} \oplus \mathcal{N}_{\theta_{2}, M}}\left(N_{1}\right)+\Pi^{\mathcal{N}_{\theta_{1}, M} \oplus \mathcal{N}_{\theta_{2}, M}}\left(N_{2}\right)=N_{1}+\Pi^{\mathcal{N}_{\theta_{1}, M} \oplus \mathcal{N}_{\theta_{2}, M}}\left(N_{2}\right)$ by linearity and the property that $\Pi^{M}(x)=x$ for $x \in M$ of the orthogonal projection. Since the two spaces $\mathcal{N}_{\theta_{1}, M}$ and $\mathcal{N}_{\theta_{2}, M}$ are orthogonal, it follows that $\Pi^{\mathcal{N}_{\theta_{1}, M} \oplus \mathcal{N}_{\theta_{2}, M}}\left(N_{2}\right) \in \mathcal{N}_{\theta_{2}, M}$. This implies $\mathbb{E}\left[\Pi^{\mathcal{N}_{\theta_{1}, M} \oplus \mathcal{N}_{\theta_{2}, M}}\left(N_{2}\right) \mid \mathcal{F}_{t}\right]=0$ for $t \leq T_{1}$ and therefore

$$
\tilde{G}(\tilde{\psi})_{t}=\mathbb{E}\left[N_{1} \mid \mathcal{F}_{t}\right], \text { for } t \leq T_{1} .
$$

Comparing with (2.18) completes the proof.

The last proposition can in general not be extended for $\alpha \neq 0$, that is when $X$ is not a local martingale. We provide a counter example in Appendix A.

We have to derive the constant hedge position in $\left[T_{1}, T_{2}\right]$. The following Proposition is not new, it is shown in greater generality in (Schweizer, 1995b) but not together with the continuous hedging before $T_{1}$. Since the proof becomes rather simple in our restricted case with $X_{t}$ a martingale and only one hedging period, it is provided here. Due to the orthogonality of $\mathcal{N}_{\theta_{1}, M}$ and $\mathcal{N}_{\theta_{2}, M}$, the projection can be done separately and the problem reduces to finding $N \in \mathcal{N}_{\theta_{2}, M}$ such that $\mathbb{E}\left[\left(H-V_{0}-N\right)^{2}\right]$ is minimized.

Proposition 2.7. Let $H$ be an $\mathcal{F}_{T_{2}}$-measurable payoff with $\mathbb{E}\left[H^{2}\right]<\infty$ and, as before $V_{T_{1}}$ the portfolio value at time $T_{1}$. Let further $M$ be a martingale. Then, the optimal $\mathcal{F}_{T_{1}-\text {-measurable }}$ 
strategy $\psi_{T_{1}}$ to minimize

$$
\min _{\zeta} \mathbb{E}\left[\left(H-V_{T_{1}}-\zeta\left(M_{T_{2}}-M_{T_{1}}\right)\right)^{2}\right]
$$

is given by:

$$
\psi_{T_{1}}=\frac{\mathbb{E}\left[(H)\left(M_{T_{2}}-M_{T_{1}}\right) \mid \mathcal{F}_{T_{1}-}\right]}{\mathbb{E}\left[\left(M_{T_{2}}-M_{T_{1}}\right)^{2} \mid \mathcal{F}_{T_{1}-}\right]} .
$$

Especially the strategy $\psi_{T_{1}}$ does not depend on the initial capital $V_{T_{1}}$. The optimal $\mathcal{F}_{T_{1}}$ measurable initial capital $V_{T_{1}}$ is then given by $V_{T_{1}}=\mathbb{E}\left[H \mid \mathcal{F}_{T_{1}}\right]$, which can be guaranteed in the case where the market is complete without the trading restrictions.

Proof. We note that for $L^{H-V_{T_{1}}}:=H-V_{T_{1}}-\Pi^{\mathcal{N}_{\theta_{2}, M}}\left(H-V_{T_{1}}\right), L^{H-V_{T_{1}}} \perp \Pi^{\mathcal{N}_{\theta_{2}, M}}\left(H-V_{T_{1}}\right)$. We try to find directly an element $N=\psi_{T_{1}}\left(M_{T_{2}}-M_{T_{1}}\right) \in \mathcal{N}_{\theta_{2}, M}$ that solves the orthogonality relation. By definition of the scalar product this means finding $\mathcal{F}_{T_{1}}$-measurable $\psi_{T_{1}}$ such that

$$
\begin{aligned}
0=\left(L^{H}, \psi_{T_{1}}\left(M_{T_{2}}-M_{T_{1}}\right)\right) & =\left(H-V_{0}-\psi_{T_{1}}\left(M_{T_{2}}-M_{T_{1}}\right), \psi_{T_{1}}\left(M_{T_{2}}-M_{T_{1}}\right)\right) \\
& =\mathbb{E}\left[\left(H-V_{T_{1}}-\psi_{T_{1}}\left(M_{T_{2}}-M_{T_{1}}\right)\right)\left(M_{T_{2}}-M_{T_{1}}\right)\right]
\end{aligned}
$$

By the tower law this is certainly fulfilled if the conditional expectation is zero. We find

$$
\begin{aligned}
0 & =\mathbb{E}\left[\left(H-V_{T_{1}}-\psi_{T_{1}}\left(M_{T_{2}}-M_{T_{1}}\right)\right)\left(M_{T_{2}}-M_{T_{1}}\right) \mid \mathcal{F}_{T_{1}}\right] \\
& =\mathbb{E}\left[\left(H-V_{T_{1}}\right)\left(M_{T_{2}}-M_{T_{1}}\right) \mid \mathcal{F}_{T_{1}}\right]-\psi_{T_{1}} \mathbb{E}\left[\left(M_{T_{2}}-M_{T_{1}}\right)^{2} \mid \mathcal{F}_{T_{1}}\right] \\
& =\mathbb{E}\left[H\left(M_{T_{2}}-M_{T_{1}}\right) \mid \mathcal{F}_{T_{1}}\right]-\psi_{T_{1}} \mathbb{E}\left[\left(M_{T_{2}}-M_{T_{1}}\right)^{2} \mid \mathcal{F}_{T_{1}}\right],
\end{aligned}
$$

where the last equality stems from the fact that $M$ is square integrable martingale and $V_{T_{1}}$ and $M_{T_{2}}-M_{T_{1}}$ are orthogonal. Solving for $\psi_{T_{1}}$ yields

$$
\psi_{T_{1}}=\frac{\mathbb{E}\left[(H)\left(M_{T_{2}}-M_{T_{1}}\right) \mid \mathcal{F}_{T_{1}}\right]}{\mathbb{E}\left[\left(M_{T_{2}}-M_{T_{1}}\right)^{2} \mid \mathcal{F}_{T_{1}}\right]}
$$

By general Hilbert space theory this minimizes the norm $\left\|\left(H-V_{T_{1}}\right)-\zeta\left(M_{T_{2}}-M_{t}\right)\right\|$ for each $V_{T_{1}}$. As in the proof of Proposition 2.4 the remaining risk is $\left\|L^{H-V_{T_{1}}}\right\|^{2}=\left\|H-V_{T_{1}}\right\|^{2}-$ $\|G(\psi)\|^{2}$. It remains to minimize $\left\|H-V_{T_{1}}\right\|^{2}$. Since the $\mathcal{F}_{T_{1}}$-measurable random variable $V_{T_{1}}=\mathbb{E}\left[H \mid \mathcal{F}_{T_{1}}\right]$ minimizes $\left\{\mathbb{E}\left[\left(H-V_{T_{1}}\right)^{2} \mid \mathcal{F}_{T_{1}}\right] \mid V_{T_{1}}\right.$ square int., $\mathcal{F}_{T_{1}}$-meas. $\}$, it minimizes $\{\| H$ $V_{T_{1}} \|^{2} \mid V_{T_{1}}$ square int., $\mathcal{F}_{T_{1}}$-meas. $\}$.

Note that in the situation where the market is incomplete even when continuous trading is allowed, we can not assure that $V_{T_{1}}=\mathbb{E}\left[H \mid \mathcal{F}_{T_{1}}\right]$.

\section{The optimal hedge in some concrete cases}

In this section we derive explicit formulas for three specific processes that are relevant in the context of energy markets. We focus our attention on claims $H$ being European put and call options on the average of the spot price over some fixed time period.

\subsection{Geometric Brownian Motion}

We calculate $\psi_{T_{1}}$ explicitly for the case where $X_{t}$ is driven by the SDE

$$
\mathrm{d} X_{t}=\sigma(t) X_{t} \mathrm{~d} W_{t}
$$


with deterministic, time dependent volatility $\sigma(t)$. This dynamics especially includes the model proposed in (Lucia and Schwartz, 2002) where $\sigma(t)=\widehat{\sigma} \exp \left(-\alpha\left(T_{2}-t\right)\right)$ for constant $\alpha$ and $\widehat{\sigma}$. The model conveniently allows to account for the Samuelson effect, that is, close to delivery the volatility of a fixed time future or a future with short delivery period increases and approaches the spot volatility.

We assume trading is continuously possible in the period $\left[0, T_{1}\right]$ but the hedge positions are constant in $\left[T_{1}, T_{2}\right]$. We know from Proposition 2.6 that the hedge positions up to time $T_{1}$ are given by the usual Black Scholes delta. The following proposition provides the hedge position after $T_{1}$.

Proposition 3.1. The hedge positions at $T_{1}$ for the call payoff $C\left(X_{T_{2}}\right)=\left(X_{T_{2}}-K\right)^{+}$when the dynamics of $X_{t}$ follows (3.1) is given by

$$
\psi_{T_{1}}^{\text {call }}=\frac{X_{T_{1}} e^{\sigma_{*}^{2}} \Phi\left(2 \sigma_{*}-\tilde{K}\right)-\left(K+X_{T_{1}}\right) \Phi\left(\sigma_{*}-\tilde{K}\right)+K \Phi(-\tilde{K})}{X_{T_{1}}\left(e^{\sigma_{*}^{2}}-1\right)}
$$

with

$$
\tilde{K}=\frac{\log \left(K / X_{T_{1}}\right)+\frac{\sigma_{*}^{2}}{2}}{\sigma_{*}}
$$

where $\sigma_{*}$ abbreviates the integrated variance $\sigma_{*}^{2}:=\int_{T_{1}}^{T_{2}} \sigma^{2}(s) \mathrm{d} s$ and $\Phi(x)$ denotes the cumulative standard normal distribution function. The hedge positions for the put payoff $P\left(X_{T_{2}}\right)=\left(X_{T_{2}}-\right.$ $K)^{-}$are given by $\psi_{T_{1}}^{\text {put }}=\psi_{T_{1}}^{\text {call }}-1$.

Proof. We calculate directly using Proposition 2.7

$$
\begin{aligned}
& \mathbb{E}\left[C\left(X_{T_{2}}\right)\left(X_{T_{2}}-X_{T_{1}}\right)\right] \\
= & \int_{-\infty}^{\infty} C\left(X_{T_{1}} e^{\frac{-\sigma_{*}^{2}}{2}+\sigma_{*} y}\right)\left(X_{T_{1}} e^{\frac{-\sigma_{*}^{2}}{2}+\sigma_{*} y}-X_{T_{1}}\right) \frac{1}{\sqrt{2 \pi}} e^{\frac{-y^{2}}{2}} \mathrm{~d} y \\
= & \int_{\tilde{K}}^{\infty} C\left(X_{T_{1}} e^{\frac{-\sigma_{*}^{2}}{2}+\sigma_{*} y}\right)\left(X_{T_{1}} e^{\frac{-\sigma_{*}^{2}}{2}+\sigma_{*} y}-X_{T_{1}}\right) \frac{1}{\sqrt{2 \pi}} e^{\frac{-y^{2}}{2}} \mathrm{~d} y
\end{aligned}
$$

since the integrand is zero for $y$ below $\tilde{K}$. To avoid too long formulas we calculate the integral over each summand in 3.3 separately. The first one becomes, using the definition of $C\left(X_{T_{2}}\right)$ :

$$
\begin{aligned}
& \int_{\tilde{K}}^{\infty}\left(X_{T_{1}} e^{\frac{-\sigma_{*}^{2}}{2}+\sigma_{*} y}-K\right) X_{T_{1}} e^{\frac{-\sigma_{*}^{2}}{2}+\sigma_{*} y} \frac{1}{\sqrt{2 \pi}} e^{\frac{-y^{2}}{2}} \mathrm{~d} y \\
= & X_{T_{1}}^{2} e^{\sigma_{*}^{2}} \int_{\tilde{K}}^{\infty} e^{-\frac{1}{2}\left(y-2 \sigma_{*}\right)^{2}} \mathrm{~d} y-K X_{T_{1}} \int_{\tilde{K}}^{\infty} e^{-\frac{1}{2}\left(y-\sigma_{*}\right)^{2}} \frac{1}{\sqrt{2 \pi}} \mathrm{d} y \\
= & X_{T_{1}}^{2} e^{\sigma_{*}^{2}} \int_{\tilde{K}-2 \sigma_{*}}^{\infty} e^{-\frac{1}{2} x^{2}} \frac{1}{\sqrt{2 \pi}} \mathrm{d} x-K X_{T_{1}} \int_{\tilde{K}-\sigma_{*}}^{\infty} e^{-\frac{1}{2} x^{2}} \frac{1}{\sqrt{2 \pi}} \mathrm{d} x
\end{aligned}
$$

and with $\Phi(x)=1-\Phi(-x),(3.4)$ can be written as:

$$
X_{T_{1}}^{2} e^{\sigma_{*}^{2}} \Phi\left(2 \sigma_{*}-\tilde{K}\right)-K X_{T_{1}} \Phi\left(\sigma_{*}-\tilde{K}\right)
$$

The second summand in $(3.3)$ can be calculated similarly and becomes

$$
-X_{T_{1}}^{2} \Phi\left(\sigma_{*}-\tilde{K}\right)+K X_{T_{1}} \Phi(-\tilde{K}) .
$$

Altogether this yields 3.2 with $\mathbb{E}\left[\left(X_{T_{2}}-X_{T_{1}}\right)^{2}\right]=X_{T_{1}}^{2}\left(e^{\sigma_{*}^{2}}-1\right)$. 

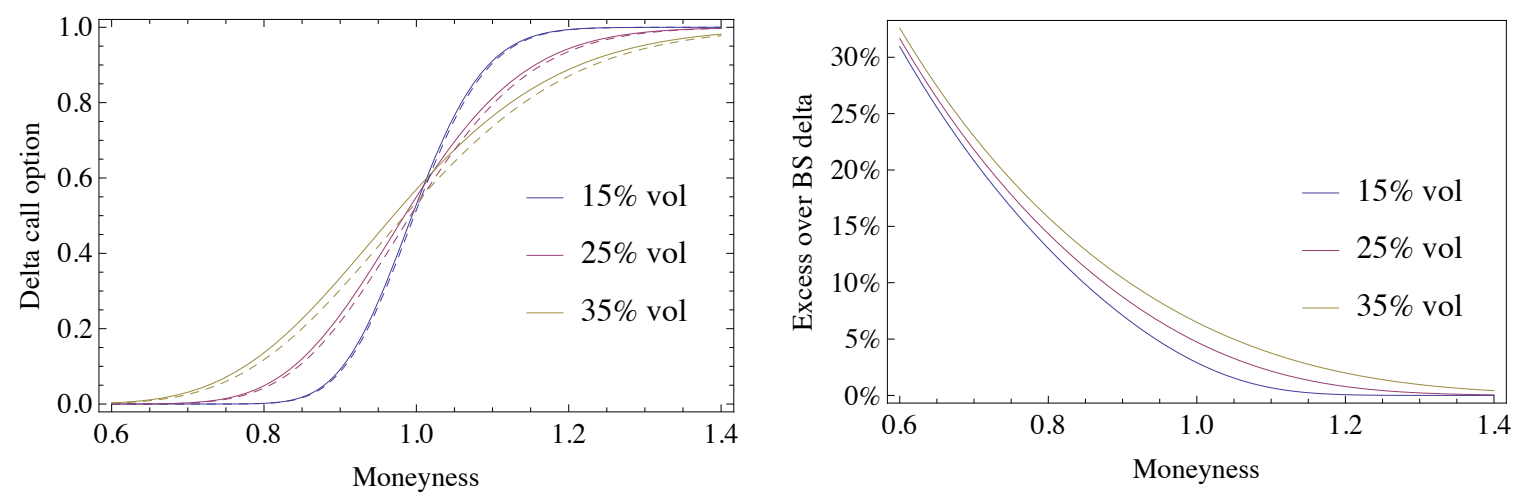

Figure 1: Left: Black Scholes delta(dashed) and the discrete quadratic hedge position for a call option with three month discrete period. Right: Percentage excess of the quadratic hedging delta compared to Black Scholes delta.

Since $X_{T_{2}}-K=C\left(X_{T_{2}}\right)-P\left(X_{T_{2}}\right)$ and

$$
\frac{\mathbb{E}\left[\left(X_{T_{2}}-K\right)\left(X_{T_{2}}-X_{T_{1}}\right)\right]}{\mathbb{E}\left[\left(X_{T_{2}}-X_{T_{1}}\right)^{2}\right]}=\frac{\mathbb{E}\left[X_{T_{2}}\left(X_{T_{2}}-X_{T_{1}}\right)\right]}{\mathbb{E}\left[\left(X_{T_{2}}-X_{T_{1}}\right)^{2}\right]}=1
$$

due to the martingale property of $X$, the linearity of the projection implies $\psi_{T_{1}}^{\text {put }}=\psi_{T_{1}}^{\text {call }}-1$.

In Figure 1 we present both, the Black Scholes delta and the discrete quadratic hedging delta under the model (3.1) with three different levels of constant volatility. The payoff is a call option with fixed time to maturity of 3 month. The x-axis shows the moneyness of the option in both plots. In the left figure the y-axis shows the hedge positions as absolute numbers, in the right figure it shows the percentage excess of the quadratic hedging delta over the Black-Scholes delta. While in the left figure the discrete hedging delta appears to be only slightly above the normal Black-Scholes delta, the right figure shows that the relative difference can become rather large.

\subsection{Stochastic volatility}

As a second example we consider the Barndorff-Nielsen and Shephard stochastic volatility model proposed in (Barndorff-Nielsen and Shephard, 2001) and considered for commodity markets in (Benth, 2011). Assume that the future price is driven by the SDE

$$
\mathrm{d} X_{t}=X_{t} \sigma_{t} \mathrm{~d} B_{t}
$$

with stochastic volatility $Y_{t}=\sigma_{t}^{2}$ driven by

$$
\mathrm{d} Y_{t}=-\lambda_{t} Y_{t} \mathrm{~d} t+\mathrm{d} L_{t}
$$

with $L_{t}$ a finite activity subordinator process (increasing Lévy process) without deterministic drift and with Lévy measure $w(x)$ such that the cumulant transform $\kappa(\theta)=\log \mathbb{E}\left[e^{\theta L_{1}}\right]$, exists for $\theta \in[-b, b]$ and is given by

$$
\kappa(\theta)=\int_{\mathbb{R}_{+}}\left(e^{\theta x}-1\right) w(x) \mathrm{d} x .
$$


We assume $\lambda_{t}$ to be deterministic, positive and in $\mathcal{C}\left(\left[0, T_{2}\right]\right.$. Note that as $L$ is a pure-jump Lévy process, it will be independent of $B$, and thus the stochastic volatility $\sigma$ is independent of $B$.

First note that by applying Itô we get

$$
Y_{u}=Y_{t} e^{-\int_{t}^{u} \lambda_{r} \mathrm{~d} r}+\int_{t}^{u} e^{-\int_{s}^{u} \lambda_{r} \mathrm{~d} r} \mathrm{~d} L_{s}
$$

Integrating over the time interval $[t, T]$ yields

$$
\int_{t}^{T} \sigma_{u}^{2} \mathrm{~d} u=Y_{t} \int_{t}^{T} e^{-\int_{t}^{u} \lambda_{r} \mathrm{~d} r} \mathrm{~d} u+\int_{t}^{T} \int_{t}^{u} e^{-\int_{s}^{u} \lambda_{r} \mathrm{~d} r} \mathrm{~d} L_{s} \mathrm{~d} u
$$

Using the stochastic Fubini Theorem (Protter, 2005, Thm. 65) we obtain for the integrated variance

$$
\int_{t}^{T} \sigma_{u}^{2} \mathrm{~d} u=\sigma_{t}^{2} \epsilon(t, T)+\int_{t}^{T} \epsilon(u, T) \mathrm{d} L_{u}
$$

with continuous function $\epsilon(s, T)$.

This representation for the integrated variance allows us to derive the Laplace transform of the distribution of the $\log$ price process $Z_{T}:=\ln X_{T}=\ln X_{0}-\frac{1}{2} \int_{0}^{T} \sigma_{s}^{2} \mathrm{~d} s+\int_{0}^{T} \sigma_{s} \mathrm{~d} B_{s}$ given the information up to time $T$. For constant $\lambda$ the following theorem is proved in (Nicolato and Venardos, 2003) and extends with only minor changes to our model setup.

Lemma 3.2. The Laplace transform $\phi\left(t, T, z, \sigma_{t}\right)=\mathbb{E}\left[\exp \left\{z\left(Z_{T}-Z_{t}\right) \mid \sigma_{t}\right\}\right]$ is given by

$$
\phi\left(t, T, z, \sigma_{t}\right)=\exp \left\{\left(\frac{1}{2} z^{2}-z \frac{1}{2}\right) \sigma_{t}^{2} \epsilon(t, T)+\int_{t}^{T} \kappa(f(s, z)) \mathrm{d} s\right\}
$$

with $f(s, z):=\left(\frac{1}{2} z^{2}-z \frac{1}{2}\right) \epsilon(s, T)$ and $\kappa(\theta)$ as in (3.7). The transform is well defined in the open stripe $\mathcal{S}=\left\{z \in \mathbb{C}: \Re(z) \in\left(\theta_{-}, \theta_{+}\right\}\right.$with

$$
\begin{aligned}
& \theta_{+}=\frac{1}{2}+\sqrt{\frac{1}{4}+2 b \epsilon(T, T)^{-1}} \\
& \theta_{-}=-\frac{1}{2}-\sqrt{\frac{1}{4}+2 b \epsilon(t, T)^{-1}}
\end{aligned}
$$

Proof. see (Nicolato and Venardos, 2003, Thm. 2.2.)

We use $\phi\left(t, T, z, \sigma_{t}\right)$ to derive an integral representation for the prices and hedge positions for calls and puts. For this we need the (bilateral) Laplace transform defined by:

$$
\mathcal{L}\{f(\cdot)\}(z)=\int_{-\infty}^{\infty} e^{-z t} f(t) \mathrm{d} t .
$$

For calls and puts the following Lemma provides the Laplace transform of their payoff and modified payoff functions, which we will need in the following.

Lemma 3.3. Let

$$
\begin{aligned}
& L_{1}(z)=\frac{X_{t}}{z(z-1)}\left(\frac{K}{X_{t}}\right)^{(1-z)} \\
& L_{2}(z)=-\frac{X_{T_{1}}^{2}}{2-z}\left(\frac{K}{X_{T_{1}}}\right)^{(2-z)}+\frac{\left(K X_{T_{1}}+X_{T_{1}}^{2}\right)}{1-z}\left(\frac{K}{X_{T_{1}}}\right)^{(1-z)}+\frac{K X_{T_{1}}}{z}\left(\frac{K}{X_{T_{1}}}\right)^{-z}
\end{aligned}
$$


and

$$
\begin{aligned}
& c_{1}(y)=\left(X_{t} e^{y}-K\right)^{+} \\
& p_{1}(y)=\left(K-X_{t} e^{y}\right)^{+} \\
& c_{2}(y)=\left(X_{T_{1}} e^{y}-K\right)^{+}\left(X_{T_{1}} e^{y}-X_{T_{1}}\right) \\
& p_{2}(y)=\left(K-X_{T_{1}} e^{y}\right)^{+}\left(X_{T_{1}} e^{y}-X_{T_{1}}\right)
\end{aligned}
$$

Then

$$
\begin{aligned}
& L_{1}(z)= \begin{cases}\mathcal{L}\left\{c_{1}(\cdot)\right\}(z) & \text { for } z \text { with } \Re(z)>1 \\
\mathcal{L}\left\{p_{1}(\cdot)\right\}(z) & \text { for } z \text { with } \Re(z)<0,\end{cases} \\
& L_{2}(z)= \begin{cases}\mathcal{L}\left\{c_{2}(\cdot)\right\}(z) & \text { for } z \text { with } \Re(z)>2 \\
\mathcal{L}\left\{p_{2}(\cdot)\right\}(z) & \text { for } z \text { with } \Re(z)<-2,\end{cases}
\end{aligned}
$$

especially the bilateral Laplace transform is convergent in the respective domain.

Proof. See appendix

We can use the representation of the Laplace transforms in the previous Lemma to derive an integral representation of the hedge positions for put and call payoffs.

Proposition 3.4. Let $f\left(X_{T_{1}} e^{y}\right)$ be a payoff function such that the Laplace transform of the modified payoff function

$$
g(y):=f\left(X_{T_{1}} e^{y}\right)\left(X_{T_{1}} e^{y}-X_{T_{1}}\right)
$$

is well defined in a stripe $\mathcal{R}:=\left\{z \in \mathbb{C}: b_{1} \leq \Re(z) \leq b_{2}\right\}$ and $\mathcal{S} \cap \mathcal{R} \neq \emptyset$ with $\mathcal{S}$ as defined in Lemma 3.2 and of finite variation on compacts. Then the discrete hedge positions for the model (3.5) in the time period $\left[T_{1}, T_{2}\right]$ are given by

$$
\psi_{T_{1}}=\frac{\frac{1}{2 \pi i} \int_{c-i \infty}^{c+i \infty} \mathcal{L}\{g(\cdot)\}(z) \phi\left(T_{1}, T_{2}, z, \sigma_{T_{1}}\right) \mathrm{d} z}{\mathbb{E}\left[\left(X_{T_{2}}-X_{T_{1}}\right)^{2} \mid \mathcal{F}_{T_{1}}\right]}
$$

with

$$
\mathbb{E}\left[\left(X_{T_{2}}-X_{T_{1}}\right)^{2} \mid \mathcal{F}_{T_{1}}\right]=X_{t}^{2}\left(e^{\sigma_{t}^{2} \epsilon\left(t, T_{2}\right)} e^{\int_{t}^{T_{2}} \kappa\left(\epsilon\left(s, T_{2}\right)\right) \mathrm{d} s}-1\right),
$$

and $\epsilon(t, T)$ and $\kappa(\theta)$ as defined in $(3.7)$.

Proof. We know from Proposition 2.7 that $\psi_{T_{1}}=\frac{\mathbb{E}\left[H\left(X_{T_{2}}-X_{T_{1}}\right) \mid \mathcal{F}_{T_{1}}\right]}{\mathbb{E}\left[\left(X_{T_{2}}-X_{T_{1}}\right)^{2} \mid \mathcal{F}_{T_{1}}\right]}$. We first calculate the denominator: For this purpose we introduce the filtration

$$
\mathcal{G}_{t}:=\sigma\left\{\sigma^{2}, 0 \leq s \leq T_{2}\right\} \bigvee \mathcal{F}_{t}
$$

and recall that $\sigma^{2}$ is independent of $B$. First note that due to the martingale property we obtain

$$
\mathbb{E}\left[\left(X_{T_{2}}-X_{T_{1}}\right)^{2} \mid \mathcal{F}_{T_{1}}\right]=\mathbb{E}\left[X_{T_{2}}^{2} \mid \mathcal{F}_{T_{1}}\right]-X_{T_{1}}^{2}
$$


We have $X_{T_{2}}^{2}=X_{T_{1}}^{2} e^{-\int_{T_{1}}^{T_{2}} \sigma_{s}^{2} \mathrm{~d} s+\int_{T_{1}}^{T_{2}} 2 \sigma_{s} \mathrm{~d} B_{s}}$ and therefore

$$
\begin{aligned}
\mathbb{E}\left[X_{T_{2}}^{2} \mid \mathcal{F}_{T_{1}}\right] & =X_{T_{1}}^{2} \mathbb{E}\left[e^{-\int_{T_{1}}^{T_{2}} \sigma_{s}^{2} \mathrm{~d} s+\int_{T_{1}}^{T_{2}} 2 \sigma_{s} \mathrm{~d} B_{s}} \mid \mathcal{F}_{T_{1}}\right] \\
& =X_{T_{1}}^{2} \mathbb{E}\left[e^{-\int_{T_{1}}^{T_{2}} \sigma_{s}^{2} \mathrm{~d} s} \mathbb{E}\left[e^{\int_{T_{1}}^{T_{2}} 2 \sigma_{s} \mathrm{~d} B_{s}} \mid \mathcal{G}_{T_{1}}\right] \mid \mathcal{F}_{T_{1}}\right] \\
& =X_{T_{1}}^{2} \mathbb{E}\left[e^{\int_{T_{1}}^{T_{2}} \sigma_{s}^{2} \mathrm{~d} s} \mid \mathcal{F}_{T_{1}}\right]
\end{aligned}
$$

Using 3.9 we receive

$$
\mathbb{E}\left[X_{T_{2}}^{2} \mid \mathcal{F}_{T_{1}}\right]=X_{T_{1}}^{2} e^{\sigma_{t}^{2} \epsilon\left(T_{1}, T_{2}\right)} \mathbb{E}\left[e^{\int_{T_{1}}^{T_{2}} \epsilon\left(s, T_{2}\right) d L_{s}} \mid \mathcal{F}_{T_{1}}\right]
$$

Since the function $\epsilon(s, T)$ is continuous in $s$ and bounded it follows from (Eberlein and Raible, 1999, Lemma 3.1.) that

$$
\mathbb{E}\left[e^{\int_{T_{1}}^{T_{2}} \epsilon\left(s, T_{2}\right) \mathrm{d} L_{s}} \mid \mathcal{F}_{T_{1}}\right]=e^{\int_{T_{1}}^{T_{2}} \kappa\left(\epsilon\left(s, T_{2}\right)\right) \mathrm{d} s}
$$

with $\epsilon(t, T)$ and $\kappa(\theta)$ as defined in 3.7 . 5 From (3.16) together with (3.13, Equation 3.11) follows.

To calculate the numerator of the quantity in Proposition 2.7. we choose $c \in \mathcal{S} \cap \mathcal{R}$. We want to calculate

$$
\mathbb{E}\left[f\left(X_{T_{1}} e^{Z_{T_{2}}-Z_{T_{1}}}\right)\left(X_{T_{1}} e^{Z_{T_{2}}-Z_{T_{1}}}-X_{T_{1}}\right) \mid \mathcal{F}_{T_{1}}\right]=\mathbb{E}\left[g\left(Z_{T_{2}}-Z_{T_{1}}\right) \mid \mathcal{F}_{T_{1}}\right]
$$

with $g(y)$ as defined in (3.10). Since $g(y)$ is of bounded variation on compacts and $\mathcal{L}\{g(\cdot)\}(z)$ is well defined for $\Re(z)=c$, we can use the Laplace inversion theorem (Widder, 1946, Chapter 6 , Theorem 5a) to retrieve $g(y)$ by integrating along the vertical line going through $c$ :

$$
g(y)=\frac{1}{2 \pi i} \int_{c-i \infty}^{c+i \infty} \mathcal{L}\{g(\cdot)\}(z) e^{z y} \mathrm{~d} z
$$

Using this expression we can calculate $\mathbb{E}\left[g\left(Z_{T_{2}}-Z_{T_{1}}\right) \mid \mathcal{F}_{T_{1}}\right]$ by remembering that the line of integration lies within the set $\mathcal{S}$, and by changing the order of integration

$$
\begin{aligned}
\mathbb{E}\left[g\left(Z_{T_{2}}-Z_{T_{1}}\right) \mid \mathcal{F}_{T_{1}}\right] & =\frac{1}{2 \pi i} \mathbb{E}\left[\int_{c-i \infty}^{c+i \infty} \mathcal{L}\{g(\cdot)\}(z) e^{z\left(Z_{T_{2}}-Z_{T_{1}}\right.} \mathrm{d} z \mid \mathcal{F}_{T_{1}}\right] \\
& =\frac{1}{2 \pi i} \int_{c-i \infty}^{c+i \infty} \mathcal{L}\{g(\cdot)\}(z) \mathbb{E}\left[e^{z\left(Z_{T_{2}}-Z_{T_{1}}\right)} \mid \mathcal{F}_{T_{1}}\right] \mathrm{d} z \\
& =\frac{1}{2 \pi i} \int_{c-i \infty}^{c+i \infty} \mathcal{L}\{g(\cdot)\}(z) \phi\left(T_{1}, T_{2}, z, \sigma_{T_{1}}\right) \mathrm{d} z,
\end{aligned}
$$

with $\phi\left(s, T, z, \sigma_{s}\right)$ being the Laplace transform of the incremental log price density as derived in Lemma 3.2 .

This especially allows us to derive integral formulas for the hedge positions of put and call payoffs as Lemma 3.3 provides the Laplace transform of their modified payoff function.

It remains to calculate the hedge positions in the continuous trading period. First we prove certain smoothness properties of the pricing functions for put and call payoffs, which we will

\footnotetext{
${ }^{5}$ In (Eberlein and Raible 1999 , Lemma 3.1.) the integral starts at 0 and there is no conditional expectation. It can easily be seen that the reasoning in the proof works the same way with the conditional expectation.
} 
need to derive the continuous hedge positions.

Lemma 3.5. The prices under the model (3.5) of the call and put option with payout (2.3) and (2.4) can be written as a function of $t, X_{t}$ and $\sigma_{t}^{2}$ such that $C\left(t, X_{t}, \sigma_{t}^{2}\right)=\mathbb{E}\left[C\left(X_{T_{2}}\right) \mid \mathcal{F}_{t}\right]$ and $P\left(t, X_{t}, \sigma_{t}^{2}\right)=\mathbb{E}\left[P\left(X_{T_{2}}\right) \mid \mathcal{F}_{t}\right]$, respectively. Additionally, both functions are in $\mathcal{C}^{2}\left(\left[0, T_{2}\right) \times\right.$ $\left.(0, \infty)^{2}\right)$.

Proof. See appendix.

The next proposition provides the hedge positions for the time period before $T_{1}$.

Proposition 3.6. Let the payoff function $f\left(X_{T_{2}}\right)$ be Lipschitz continuous with respect to $X_{T_{2}}$ and $f\left(t, X_{t}, \sigma_{t}^{2}\right)=\mathbb{E}\left[f\left(X_{T_{2}}\right) \mid \mathcal{F}_{t}\right]$ in $\mathcal{C}^{2}\left(\left(\left[0, T_{2}\right) \times(0, \infty)^{2}\right)\right)$. Then the hedge positions for the stochastic volatility model (3.5) without trading restrictions are given by

$$
\frac{\partial f\left(t, X_{t}, \sigma_{t}^{2}\right)}{\partial X_{t}}
$$

Proof. Note that $\left[X, \sigma^{2}\right]=0$ since $\sigma^{2}$ is quadratic pure jump process and $X$ continuous (cf. (Protter, 2005, p. 75 Theorem 28). An application of the multi-dimensional Itô formula for semimartingales (see for example (Protter, 2005, p. 81 Theorem 33)), considering that $\left[X, \sigma^{2}\right]=$ 0 and the fact that the continuous part of the quadratic variation $\left[\sigma^{2}, \sigma^{2}\right]$ is zero, yields

$$
\begin{aligned}
f\left(t, X_{t}, \sigma_{t}^{2}\right)-f\left(0, X_{0}, \sigma_{0}^{2}\right) & =\int_{0}^{t} \frac{\partial f\left(s, X_{s}, \sigma_{s-}^{2}\right)}{\partial s} \mathrm{~d} s+\int_{0}^{t} \frac{\partial f\left(s, X_{s}, \sigma_{s-}^{2}\right)}{\partial X_{s}} \mathrm{~d} X_{s} \\
& +\int_{0}^{t} \frac{\partial f\left(s, X_{s}, \sigma_{s-}^{2}\right)}{\partial \sigma_{s}^{2}} \mathrm{~d} \sigma_{s}^{2}+\frac{1}{2} \int_{0}^{t} \frac{\partial^{2} f\left(s, X_{s}, \sigma_{s-}^{2}\right)}{\partial^{2} X_{s}} X_{s}^{2} \sigma_{s}^{2} \mathrm{~d} s \\
& +\sum_{0<s \leq t}\left\{f\left(s, X_{s}, \sigma_{s}^{2}\right)-f\left(s, X_{s}, \sigma_{s-}^{2}\right)-\frac{\partial f\left(s, X_{s}, \sigma_{s-}^{2}\right)}{\partial \sigma_{s}^{2}} \Delta \sigma_{s}^{2}\right\}
\end{aligned}
$$

Denote by $\tilde{M}(\mathrm{~d} s \mathrm{~d} y)$ the compensated Poisson measure of the pure jump process $L$ and by $\mu(\mathrm{d} s \mathrm{~d} y)$ its Lévy measure. Together with the definition of $\mathrm{d} \sigma_{t}^{2}$ we can rewrite 3.20 as

$$
\begin{aligned}
f\left(t, X_{t}, \sigma_{t}^{2}\right)-f\left(0, X_{0}, \sigma_{0}^{2}\right) & =\int_{0}^{t} \frac{\partial f\left(s, X_{s}, \sigma_{s-}^{2}\right)}{\partial s} \mathrm{~d} s+\int_{0}^{t} \frac{\partial f\left(s, X_{s}, \sigma_{s-}^{2}\right)}{\partial X_{s}} \mathrm{~d} X_{s} \\
& -\int_{0}^{t} \frac{\partial f\left(s, X_{s}, \sigma_{s-}^{2}\right)}{\partial \sigma_{s}^{2}} \lambda_{s} \sigma_{s}^{2} \mathrm{~d} s+\frac{1}{2} \int_{0}^{t} \frac{\partial^{2} f\left(s, X_{s}, \sigma_{s-}^{2}\right)}{\partial^{2} X_{s}} X_{s}^{2} \sigma_{s}^{2} \mathrm{~d} s \\
& +\int_{0}^{t} \int_{0}^{\infty}\left(f\left(s, X_{s}, \sigma_{s}^{2}\right)-f\left(s, X_{s}, \sigma_{s-}^{2}\right) \tilde{M}\left(\mathrm{~d} s \mathrm{~d} \sigma^{2}\right)\right. \\
& +\int_{0}^{t} \int_{0}^{\infty}\left(f\left(s, X_{s}, \sigma_{s}^{2}\right)-f\left(s, X_{s}, \sigma_{s-}^{2}\right) \mu\left(\mathrm{d} s \mathrm{~d} \sigma^{2}\right) .\right.
\end{aligned}
$$

The $\mathrm{d} s$ terms and the $\mu\left(\mathrm{d} s \mathrm{~d} \sigma^{2}\right)$ integral have to vanish due to the martingale property of the price process and we obtain:

$$
\begin{aligned}
f\left(t, X_{t}, \sigma_{t}^{2}\right)-f\left(0, X_{0}, \sigma_{0}^{2}\right) & =\int_{0}^{t} \frac{\partial f\left(s, X_{s}, \sigma_{s-}^{2}\right)}{\partial X_{s}} \mathrm{~d} X_{s} \\
& +\int_{0}^{t} \int_{0}^{\infty}\left(f\left(s, X_{s}, \sigma_{s}^{2}\right)-f\left(s, X_{s}, \sigma_{s-}^{2}\right) \tilde{M}(\mathrm{~d} s \mathrm{~d} y)\right.
\end{aligned}
$$


Note that if the payoff is Lipschitz, the same holds for $f\left(t, x, \sigma_{t}^{2}\right)$ since for Lipschitz constant $C$ we find

$$
\begin{aligned}
\left|f\left(t, x, \sigma_{t}^{2}\right)-f\left(t, y, \sigma_{t}^{2}\right)\right| \leq \mathbb{E}\left[f\left(x e^{Z_{T}-Z_{t}}\right)-f\left(y e^{Z_{T}-Z_{t}}\right)\right] & \leq C|x-y| \mathbb{E}\left[e^{Z_{T}-Z_{t}}\right] \\
& =C|x-y|,
\end{aligned}
$$

where the last step is due to the martingale property. The portfolio process $V_{t}=\int_{0}^{t} \psi_{s} \mathrm{~d} X_{s}$ is a martingale by the choice of $\psi_{t}$ and by the Lipschitz property and the square integrability of $X_{t}$, the jump integral in 3.24 is also a martingale. This, together with $V_{0}=f\left(0, X_{0}, \sigma_{0}^{2}\right)$ and again $\left[X, \sigma^{2}\right]=0$ implies that ${ }^{6}$

$$
\begin{aligned}
& \mathbb{E}\left[\left(f\left(t, X_{t}, \sigma_{t}^{2}\right)-V_{0}\right)^{2}\right]=\mathbb{E}\left[\left(\int_{0}^{t}\left(\frac{\partial f\left(s, X_{s}, \sigma_{s-}^{2}\right)}{\partial X_{s}}-\psi_{s}\right) \mathrm{d} X_{s}\right)^{2}\right] \\
+ & \mathbb{E}\left[\left(\int_{0}^{t} \int_{0}^{\infty}\left(f\left(s, X_{s}, \sigma_{s}^{2}\right)-f\left(s, X_{s}, \sigma_{s-}^{2}\right) \tilde{M}(\mathrm{~d} s \mathrm{~d} y)\right)^{2}\right] .\right.
\end{aligned}
$$

Then due to the Itô Isometry

$$
\begin{aligned}
\mathbb{E}\left[\left(f\left(t, X_{t}, \sigma_{t}^{2}\right)-V_{0}\right)^{2}\right] & =\mathbb{E}\left[\int_{0}^{t}\left(\frac{\partial f\left(s, X_{s}, \sigma_{s-}^{2}\right)}{\partial X_{s}}-\psi_{s}\right)^{2} \mathrm{~d}[X, X]_{s}\right] \\
& +\mathbb{E}\left[\int_{0}^{t} \int_{0}^{\infty}\left(f\left(s, X_{s}, \sigma_{s}^{2}\right)-f\left(s, X_{s}, \sigma_{s-}^{2}\right)^{2} \mu(\mathrm{d} s \mathrm{~d} y)\right]\right.
\end{aligned}
$$

Clearly, this expression is minimized by choosing $\psi_{s}=\frac{\partial f\left(s, X_{s}, \sigma_{s-}^{2}\right)}{\partial X_{s}}$.

In particular, the last proposition provides us with the hedge position for the put and call payoff we are interested in. We collect this in a Corollary.

Corollary 3.7. The continuous hedge position for put and call payoffs are given by

$$
\begin{aligned}
& \frac{\partial C\left(s, X_{s}, \sigma_{s-}^{2}\right)}{\partial X_{s}}=\frac{1}{2 \pi i} \int_{c-i \infty}^{c+i \infty} \frac{1}{(z-1)}\left(\frac{K}{X_{s}}\right)^{(1-z)} \phi\left(s, T, z, \sigma_{s}\right) \mathrm{d} z, c>1 \\
& \frac{\partial P\left(s, X_{s}, \sigma_{s-}^{2}\right)}{\partial X_{s}}=\frac{1}{2 \pi i} \int_{c-i \infty}^{c+i \infty} \frac{1}{(z-1)}\left(\frac{K}{X_{s}}\right)^{(1-z)} \phi\left(s, T, z, \sigma_{s}\right) \mathrm{d} z, c<0
\end{aligned}
$$

with $\phi\left(s, T, z, \sigma_{s}\right)$ as defined in Lemma 3.2.

Proof. Clearly, the payoffs fulfill the Lipschitz properties. Lemma 3.5 provides the functional form and the $\mathcal{C}^{2}$ property, such that we can apply Proposition 3.6 . For the integral representation, we can find a representation for the prices $C\left(s, X_{s}, \sigma_{s-}^{2}\right)$ and $P\left(s, X_{s}, \sigma_{s-}^{2}\right)$ by the same means as used in Proposition 3.4 by using the transform $L_{1}(z)$ as provided in Lemma 3.2 . Differentiating below the integral sign shows (3.25) and (3.25).

\subsection{Exponential additive models}

As a third example we consider the class of models where the log price process is driven by an additive process. These processes conveniently allow to account for the non-stationarity of the

\footnotetext{
${ }^{6}$ Note that for integrands $H, K$ we have that from $\left[X, \sigma^{2}\right]_{s}=0, \forall s \leq t$ it follows that $\left[H \cdot X, K \cdot \sigma^{2}\right]_{t}=0$. If in addition $H \cdot X$ and $K \cdot \sigma^{2}$ martingales, then it follows by integration by parts that $\mathbb{E}\left[(H \cdot X)\left(K \cdot \sigma^{2}\right)\right]=0(\mathrm{cf}$. (Protter, 2005, p. 75 Theorem 29)
} 
future dynamics in energy markets, and has been applied by (Goutte et al., 2014). We shall recall the definition of additive processes. For a thorough introduction see (Sato, 1999).

Definition 3.8. A stochastic process $\left(Y_{t}\right)_{0 \geq t}$ on $\mathbb{R}$ is called additive process if it satisfies the following conditions:

1. $\mathbb{P}\left(\left(Y_{0}=0\right)=0\right)$.

2. Independent increments: For an increasing sequence of times $t_{0}, \ldots, t_{n}$ the random variables $Y_{t_{0}}, Y_{t_{1}}-Y_{t_{0}}, \ldots, Y_{t_{n}}-Y_{t_{n-1}}$ are independent.

3. Stochastic continuity: $\forall \epsilon>0, \lim _{h \rightarrow 0}\left(\mathbb{P}\left(\left|\left(Y_{t+h}-Y_{t}\right)\right|\right) \geq \epsilon\right)=0$.

4. $\left(Y_{t}\right)$ has a càdlàg version.

Examples of additive processes are diffusion processes with time dependent volatility or time inhomogeneous jump diffusions. It turns out that for additive processes $\left(Y_{t}\right)_{0 \geq t}$ for every $t$ the distribution of $Y_{t}$ is infinitely divisible (see (Sato, 1999, Thm 9.1.)). This implies that there is a Lévy-Khinchine representation for the characteristic function $\phi(z)=\mathbb{E}\left[e^{z Y_{t}}\right]$ for $z=i x, x \in \mathbb{R}$. Following (Goutte et al., 2014, Proposition 3.1.) $\phi(z)$ can be extended to $z \in \mathbb{C}$ given by

$$
\begin{aligned}
\phi(z)= & \mathbb{E}\left[e^{z Y_{t}}\right]=e^{\Psi_{t}(z)} \\
& \Psi_{t}(z)=\frac{1}{2} z^{2} A_{t}+z \Gamma_{t}+\int_{[0, t] \times \mathbb{R}}\left(e^{z x}-1-z x \mathbf{1}_{|x| \leq 1}\right) \mu(\mathrm{d} s, \mathrm{~d} x)
\end{aligned}
$$

for $z$ with $\Re(z) \in \mathcal{S}:=\left\{c \in \mathbb{R} \mid \int_{[0, T] \times\{|x|>1\}} e^{c x} \mu(\mathrm{d} t, \mathrm{~d} x)<\infty\right\}$ where $A_{t}$ and $\Gamma_{t}$ are constants and $\mu(t, B)$ is given by the unique measure integrating $1 \wedge|x|^{2}$ of the Lévy-Khinchin representation for the infinitely divisible distribution of $Y_{t}$.

We assume that the asset price process is of the form

$$
X_{t}=e^{Y_{t}}
$$

where $Y$ is an additive process such that $X$ is a square integrable martingale. A special case of such a model is given by the Geometric Brownian motion example in Section 3.1, where the dynamics of $Y_{t}$ is $\mathrm{d} Y_{t}=-1 / 2 \sigma_{t}^{2} \mathrm{~d} t+\sigma_{t} \mathrm{~d} B_{t}$ with deterministic $\sigma_{t}$. The quadratic hedge positions in such a model are derived in (Goutte et al., 2014) under some assumptions on the underlying process and the payoff. We use their results to derive semi-analytic expressions for put and call prices and their continuous hedge positions in the next Proposition. Note that if $X$ defined by $X_{t}:=e^{Y_{t}}$ is a martingale, we must have that $\Psi_{t}(1)=0$.

The next proposition provides the continuous hedge positions for a call and a put payoff.

Proposition 3.9. Define the measures,

$$
\begin{aligned}
\mathrm{d} \rho_{t} & :=\mathrm{d} \Psi_{t}(2) \\
\mathrm{d} \rho_{t}(z, y) & :=\mathrm{d}\left(\Psi_{t}(z+y)-\Psi_{t}(z)-\Psi_{t}(y)\right)
\end{aligned}
$$

Denote by $\mathcal{D}$ the set $z \in \mathcal{S}$ such that

$$
\int_{0}^{T}\left|\frac{\mathrm{d} \Psi_{u}(z)}{\mathrm{d} \rho_{u}}\right| \mathrm{d} \rho_{u}<\infty
$$

Assume that the interval $z \in \mathcal{C},-2<\Re(z)<2$ is included in $\mathcal{D}$. 
Then the time-t prices for the options with payout $C\left(X_{T_{2}}\right)$ and $P\left(X_{T_{2}}\right)$ under the model (3.29) are given by

$$
\begin{aligned}
C_{t} & :=\mathbb{E}\left[C\left(X_{T_{2}}\right) \mid \mathcal{F}_{t}\right]=\frac{1}{2 \pi i} \int_{c-i \infty}^{c+i \infty} e^{\int_{t}^{T_{2}} \Psi_{s}(z) \mathrm{d} s} X_{t}^{z} \frac{K^{1-z}}{z(z-1)} \mathrm{d} z \text { for } \Re(c)>1, c \in \mathcal{D} \\
P_{t} & :=\mathbb{E}\left[P\left(X_{T_{2}}\right) \mid \mathcal{F}_{t}\right]=\frac{1}{2 \pi i} \int_{c-i \infty}^{c+i \infty} e^{\int_{t}^{T_{2}} \Psi_{s}(z) \mathrm{d} s} X_{t}^{z} \frac{K^{1-z}}{z(z-1)} \mathrm{d} z \text { for } \Re(c)<0, c \in \mathcal{D} .
\end{aligned}
$$

The hedge positions are given by:

$$
\begin{aligned}
\psi_{t}^{C} & =\frac{1}{2 \pi i} \int_{c-i \infty}^{c+i \infty} \frac{\mathrm{d} \rho_{t}(z, 1)}{\mathrm{d} \rho_{t}} e^{\int_{t}^{T_{2}} \Psi_{s}(z) \mathrm{d} s} X_{t-}^{z-1} \frac{K^{1-z}}{z(z-1)} \mathrm{d} z \text { for } \Re(c)>1, c \in \mathcal{D}, \\
\psi_{t}^{P} & =\frac{1}{2 \pi i} \int_{c-i \infty}^{c+i \infty} \frac{\mathrm{d} \rho_{t}(z, 1)}{\mathrm{d} \rho_{t}} e^{\int_{t}^{T_{2}} \Psi_{s}(z) \mathrm{d} s} X_{t-}^{z-1} \frac{K^{1-z}}{z(z-1)} \mathrm{d} z \text { for } \Re(c)<0, c \in \mathcal{D} .
\end{aligned}
$$

Proof. First note that assumption 1 and 2 on the underlying process in (Goutte et al., 2014) are automatically fulfilled if $X_{t}$ is a square integrable martingale. Additionally, we have that $\Psi_{t}(1)=0$ if $X_{t}$ is a martingale. For the two payoffs $C\left(X_{T_{2}}\right)$ and $P\left(X_{T_{2}}\right)$ we have by Lemma 3.3

$$
\begin{aligned}
& C\left(X_{T_{2}}\right)=c_{1}\left(Y_{T_{2}}\right)=\frac{1}{2 \pi i} \int_{c-i \infty}^{c+i \infty} e^{z\left(Y_{T_{2}}-Y_{t}\right)} X_{t}^{z} \frac{K^{1-z}}{z(z-1)} \mathrm{d} z \text { for } c>1 \\
& P\left(X_{T_{2}}\right)=p_{1}\left(Y_{T_{2}}\right)=\frac{1}{2 \pi i} \int_{c-i \infty}^{c+i \infty} e^{z\left(Y_{T_{2}}-Y_{t}\right)} X_{t}^{z} \frac{K^{1-z}}{z(z-1)} \mathrm{d} z \text { for } c<0,
\end{aligned}
$$

which are representations of the form $\int_{\mathbb{C}} X_{t}^{z} \Pi(\mathrm{d} z)$ where $\Pi(\mathrm{d} z)$ is some locally finite complex measure (cf. (Rudin, 1987, Chapter 6). The measure fulfills assumptions 3 in (Goutte et al., 2014 ) by the assumptions on $\mathcal{D}$. By (Goutte et al., 2014, Thm 4.1.), the time- $t$ values are given by (3.33) and (3.34). The integral in the exponential function in (Goutte et al., 2014, Thm 4.1.) contains an additional term involving $\Psi_{t}(1)$ that vanished due to the martingale property. The hedge positions are given by (3.35) and (3.36) where the definitions of $\mathrm{d} \rho_{t}(z, y)$ and $\mathrm{d} \rho_{t}$ are adapted to the martingale case.

The measure $\frac{\mathrm{d} \rho_{s}(z, 1)}{\mathrm{d} \rho_{s}}$ appearing in the integrand for the hedge positions can often be calculated in closed form (see (Goutte et al., 2014, Sec 5.1.)). Especially in the important case where $Y$ is a scaled Lévy process, i.e. $Y_{t}=\int_{0}^{t} \lambda_{s} \mathrm{~d} \Lambda_{s}$ with $\Lambda$ a Lévy process and $\lambda_{t}$ a deterministic positive function, the measure is a simple function of $\Psi_{t}(z)$. The next Proposition provides the hedge positions for the discrete period $\left[T_{1}, T_{2}\right]$.

Proposition 3.10. Let $f\left(X_{T_{1}} e^{y}\right)$ be a payoff function such that the Laplace transform of the modified payoff function

$$
g(y)=f\left(X_{T_{1}} e^{y}\right)\left(X_{T_{1}} e^{y}-X_{T_{1}}\right)
$$

is well defined in a stripe $\mathcal{R}:=\left\{z \in \mathbb{C}: b_{1} \leq \Re(z) \leq b_{2}\right\}$ and $\mathcal{S} \cap \mathcal{R} \neq \emptyset$ and $g(y)$ of finite variation on compacts. Then the discrete hedge positions for the model (3.29) in the time period $\left[T_{1}, T_{2}\right]$ are given by

$$
\psi_{T_{1}}=\frac{\frac{1}{2 \pi i} \int_{c-i \infty}^{c+i \infty} \mathcal{L}\{g(\cdot)\}(z) e^{\Psi_{T_{2}}(z)-\Psi_{T_{1}}(z)} \mathrm{d} z}{\mathbb{E}\left[\left(X_{T_{2}}-X_{T_{1}}\right)^{2} \mid \mathcal{F}_{T_{1}}\right]}
$$


for $c \in \mathcal{S} \cap \mathcal{R}$ with

$$
\mathbb{E}\left[\left(X_{T_{2}}-X_{T_{1}}\right)^{2} \mid \mathcal{F}_{T_{1}}\right]=X_{T_{1}}^{2}\left(e^{\Psi_{T_{2}}(2)-\Psi_{T_{1}}(2)}-1\right)
$$

Proof. Again, using Proposition 2.7 first we calculate the denominator of 2.19) as

$$
\begin{aligned}
\mathbb{E}\left[\left(X_{T_{2}}-X_{T_{1}}\right)^{2} \mid \mathcal{F}_{T_{1}}\right] & =X_{T_{1}}^{2} \mathbb{E}\left[e^{2\left(Y_{T_{2}}-Y_{T_{1}}\right)} \mid \mathcal{F}_{T_{1}}\right]-X_{T_{1}}^{2} \\
& =X_{T_{1}}^{2} e^{\Psi_{T_{2}}(2)-\Psi_{T_{1}}(2)}-X_{T_{1}}^{2}
\end{aligned}
$$

where $\Psi_{t}(z)$ is the cumulant generating function of $Y_{t}$ as defined in (3.27). Noting that the Laplace transform $\phi(z)$ of $Y_{T_{2}}-Y_{T_{1}}$ is given by $\phi(z)=e^{\Psi_{T_{2}}(z)-\Psi_{T_{1}}(z)}$ the rest of the proof follows exactly the proof of Proposition 3.4 .

A special case of an additive exponential model is given by $Y_{t}=\int_{0}^{t} \sigma_{s} d B_{s}-0.5 \int_{0}^{t} \sigma_{s}^{2} \mathrm{~d} s$ for deterministic $\sigma$, which is the Geometric Brownian motion model analyzed in Section 3.1. Using $\Psi_{T_{2}}(z)-\Psi_{T_{1}}(z)=1 / 2 \int_{T_{1}}^{T_{2}} \sigma_{s}^{2} \mathrm{~d} s\left(z^{2}-z\right)$ for this model and the Laplace transform $\mathcal{L}\left\{c_{2}(\cdot)\right\}(z)$ as given in Lemma 3.3, a tedious evaluation of the complex line integral in Equation 3.40 using the residue theorem brings us back to the closed form solution as shown in Proposition 3.1 .

\section{Interest rates on margin accounts}

So far we assumed that interest rates are zero and that trading gains are martingales. In actual trading at Nord Pool, the trading gains from holding a futures position accrue interest on a margin account. The portfolio process $V_{t}^{r}$ for a strategy $\psi_{t}$ including the constant instantaneous interest $r$ on the margin account is given by:

$$
V_{t}^{r}=V_{0}+\int_{0}^{t} \psi_{s} \mathrm{~d} X_{s}+\int_{0}^{t} r V_{t}^{r} \mathrm{~d} t
$$

We calculate the discounted portfolio value $R_{t} V_{t}^{r}$ with $R_{t}=e^{-r t}$ by the partial integration formula as

$$
\begin{aligned}
R_{t} V_{t}^{r} & =V_{0}+\int_{0}^{t} R_{s} \mathrm{~d} V_{s}^{r}+\int_{0}^{t} V_{s}^{r} \mathrm{~d} R_{s}+\int_{0}^{t} \mathrm{~d}\left[R, V^{r}\right]_{s} \\
& =V_{0}+\int_{0}^{t} R_{s} \psi_{s} \mathrm{~d} X_{s}+\int_{0}^{t} R_{s} r V_{s}^{r} \mathrm{~d} s+\int_{0}^{t} V_{s}^{r} \mathrm{~d} R_{s}+\int_{0}^{t} \mathrm{~d}\left[R, V^{r}\right]_{s} \\
& =V_{0}+\int_{0}^{t} R_{s} \psi_{s} \mathrm{~d} X_{s}
\end{aligned}
$$

since $\mathrm{d} R_{t}=-r R_{t} \mathrm{~d} t$ and $\mathrm{d}\left[R_{t}, V_{t}^{r}\right]=0$. From (4.1) we see that the $R_{t}$-discounted trading gain is a martingale. We obtain

$$
V_{T_{2}}^{r}=V_{0} e^{r T_{2}}+\int_{0}^{T_{2}} e^{r(T-s)} \psi_{s} \mathrm{~d} X_{s}
$$

If we were in a situation without the trading restrictions we could actually use the optimal solution $\left(V_{0}, \psi\right)$ from Proposition 2.4 and transform it by $\left(e^{-r T_{2}} V_{0}, e^{r\left(T_{2}-t\right)} \psi_{t}\right)$ to obtain a solution for the optimization including the margin account. This however does not work when we have the trading restriction for $t \in\left(T_{1}, T_{2}\right]$, because $e^{r\left(T_{2}-t\right)} \psi_{t}$ is not constant in $t \in\left(T_{1}, T_{2}\right]$. However, we can directly model the discounted margin included future process $\mathrm{d} R_{s} V_{s}^{r}=R_{s} \mathrm{~d} X_{s}$ and use investments in $R_{t} V_{t}^{r}$ as possible hedging strategies, then if we find a strategy as described 
in the last chapter to minimize

$$
\left\|R_{T_{2}} V_{T_{2}}^{r}-R_{T_{2}} H_{T_{2}}\right\|
$$

it minimizes also

$$
\left\|V_{T_{2}}^{r}-H_{T_{2}}\right\|
$$

In the next Section where we apply our results to power markets we will assume that interest rates are zero for simplicity.

\section{Application to power markets}

In this section we propose a model for the future that could be used to hedge options on energy. To state such a model is not straightforward, since due to the trading restriction, the actual price process within the time-period $\left[T_{1}, T_{2}\right]$ is unknown. We therefore start with the spot price process, that is observable at all times, and derive from it a model that fits into the class of an exponential additive model. To model the spot price we extend the Schwarz-Smith model (Schwartz and Smith, 2000) by allowing its two factors to be Lévy processes instead of Brownian motions (see (Benth et al., 2014), and (Benth and Schmeck, 2014+)). Based on the Esscher transform we find a parametric set of equivalent pricing measures for the artificial future that delivers energy at a fixed point in time. As noted in the introduction, futures traded in energy deliver over a period, not at a single time point. This future however, in contrast to the artificial future that delivers at a fixed point in time, does not follow an exponential additive process, but has a more complicated structure. We use moment matching technics to find an exponential additive process that approximates this dynamics sufficiently well. The resulting process is then covered by the results in Section 3.3. Based on spot and long dated future prices from Nord Pool, we estimate the model parameters.

We assume given a probability space $\left(\Omega, \mathbb{P},\left(\mathcal{F}_{t}\right)_{0 \leq t \leq T}\right)$ on which two dimensional Lévy process $L=\left(L^{1}, L^{2}\right)$ is defined with $L^{1}$ and $L^{2}$ being independent. We assume the spot price $S_{t}$ is modeled by

$$
\log S_{t}=\Lambda_{t}+X_{t}+Y_{t}
$$

where $\Lambda_{t}$ is a deterministic seasonality function, $X_{t}$ an Ornstein Uhlenbeck process driven by a Lévy process, i.e. $\mathrm{d} X_{t}=-\lambda X_{t} \mathrm{~d} t+\mathrm{d} L_{t}^{1}$ and $\mathrm{d} Y_{t}=\mathrm{d} L_{t}^{2}$. Let the cumulant function $\Psi(z)$ be such that $\mathbb{E}\left[e^{\left\langle z, L_{1}\right\rangle}\right]=e^{\Psi(z)}$ well defined for $z=\left(z_{1}, z_{2}\right) \in \mathbb{R}^{2},|z| \leq C$. Due to the independence of $L^{1}$ and $L^{2}$, we have $\Psi(z)=\Psi_{1}\left(z_{1}\right)+\Psi_{2}\left(z_{2}\right)$ where $\Psi_{i}$ is the cumulant for $L^{i}, i \in\{1,2\}$.

Applying the Esscher transform on the process $L$ we obtain a parametric family of equivalent measures $\mathbb{Q}_{\theta}$ where the Radon-Nikodym derivative of the measure change for $\theta \in \mathbb{R}^{2},|\theta| \leq C$ is given by:

$$
\left.\frac{\mathrm{d} \mathbb{Q}_{\theta}}{\mathrm{d} \mathbb{P}}\right|_{\mathcal{F}_{t}}=\exp \left\{\theta^{\mathrm{T}} L_{t}-t \Psi(\theta)\right\}
$$

with $\theta^{\mathrm{T}}$ denoting the transpose of the vector $\theta$. We obtain the cumulant transform under $\mathbb{Q}_{\theta}$ as $\Psi^{\theta}(z)=\Psi(z+\theta)-\Psi(\theta)$.

As already mentioned in Section 1, the future is a tradable asset and it must be a martingale, given by expectation under the measure $\mathbb{Q}_{\theta}$ :

$$
F\left(t, T_{1}, T_{2}\right)=\mathbb{E}^{\mathbb{Q}_{\theta}}\left[\frac{1}{\left(T_{2}-T_{1}\right)} \int_{T_{1}}^{T_{2}} S_{s} \mathrm{~d} s \mid \mathcal{F}_{t}\right] .
$$

We denote by $F(t, T)$ the artificial future price for delivery at a single point in time, i.e. 
$F(t, T)=\mathbb{E}\left[S_{T} \mid \mathcal{F}_{t}\right]$. Using Fubini we then obtain

$$
F\left(t, T_{1}, T_{2}\right)=\frac{1}{\left(T_{2}-T_{1}\right)} \int_{T_{1}}^{T_{2}} F(t, T) \mathrm{d} T .
$$

The following proposition provides the price process of $F(t, T)$ under $\mathbb{Q}_{\theta}$.

Proposition 5.1. The process $F(t, T)$ follows an exponential additive model under $\mathbb{Q}_{\theta}$ with time $t$ value given by:

$$
F(t, T)=\tilde{h}_{1}(T) \tilde{h}_{2}(T-t) \exp \left(\tilde{L}_{t}^{2}+e^{-\lambda T} \int_{0}^{t} e^{\lambda u} \mathrm{~d} \tilde{L}_{u}^{1}\right), \quad t \leq T
$$

with $h_{1}$ and $h_{2}$ defined as

$$
\begin{aligned}
& \tilde{h}_{1}(T)=\exp \left\{\Lambda_{T}+X_{0} e^{-\lambda T}+Y_{0}+\frac{\frac{\partial}{\partial z} \Psi_{1}^{\theta}(0)}{\lambda}\left(1-e^{-\lambda T}\right)+\frac{\partial}{\partial z} \Psi_{2}^{\theta}(0) T\right\} \\
& \tilde{h}_{2}(\tau)=\exp \left(\tau \tilde{\Psi}_{2}^{\theta}(1)+\int_{0}^{\tau} \tilde{\Psi}_{1}^{\theta}\left(e^{-\lambda u}\right) \mathrm{d} u\right)
\end{aligned}
$$

and $\tilde{L}^{i}$ defined by $\tilde{L}_{t}^{i}:=L_{t}^{i}-\frac{\partial}{\partial z} \Psi_{i}^{\theta}(0) t$ and $\tilde{\Psi}_{i}^{\theta}(z):=\Psi_{i}^{\theta}(z)-\frac{\partial}{\partial z} \Psi_{i}^{\theta}(0)$.

Proof. We find $\mathbb{E}^{\mathbb{Q}_{\theta}}\left[L_{t}^{i}\right]=\frac{\partial}{\partial z} \Psi_{i}^{\theta}(0) t$ such that $\tilde{L}_{t}^{i}=L_{t}^{i}-\frac{\partial}{\partial z} \Psi_{i}^{\theta}(0) t$ is a martingale with respect to $\mathbb{Q}_{\theta}$ with cumulant transform $\tilde{\Psi}_{i}^{\theta}$ for $i \in\{1,2\}$. The dynamics of $X_{t}$ and $Y_{t}$ under $\mathbb{Q}_{\theta}$ are therefore

$$
\begin{aligned}
\mathrm{d} X_{t} & =\left(\frac{\partial}{\partial z} \Psi_{1}^{\theta}(0)-\lambda X_{t}\right) \mathrm{d} t+\mathrm{d} \tilde{L}_{t}^{1} \\
\mathrm{~d} Y_{t} & =\frac{\partial}{\partial z} \Psi_{2}^{\theta}(0) \mathrm{d} t+\mathrm{d} \tilde{L}_{t}^{2},
\end{aligned}
$$

where $X_{t}$ has the solution

$$
X_{T}=X_{0} e^{-\lambda T}+\frac{\frac{\partial}{\partial z} \Psi_{1}^{\theta}(0)}{\lambda}\left(1-e^{-\lambda T}\right)+\int_{0}^{T} e^{\lambda(u-T)} \mathrm{d} \tilde{L}_{u}^{1}
$$

We calculate

$$
\begin{aligned}
& F(t, T)=\mathbb{E}^{\mathbb{Q}_{\theta}}\left[e^{\Lambda_{T}+X_{T}+Y_{T}} \mid \mathcal{F}_{t}\right] \\
& =\tilde{h}_{1}(T) \mathbb{E}^{\mathbb{Q}_{\theta}}\left[e^{\int_{0}^{T} \mathrm{~d} \tilde{L}_{u}^{1}+\int_{0}^{T} e^{\lambda(u-T)} \mathrm{d} \tilde{L}_{u}^{1}} \mid \mathcal{F}_{t}\right]
\end{aligned}
$$

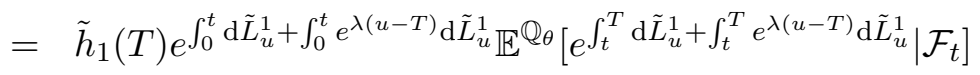

with $\tilde{h}_{1}(T)$ as defined in $(5.3)$ and where the last step is due to the measurability of the integral up to time $t$. The key formula (Eberlein and Raible, 1999. Lemma 3.1.) shows that the expectation in the last equation equals $h_{2}(T-t)$ showing (5.2).

Since $\tilde{h}_{1}(T)$ and $\tilde{h}_{2}(T-t)$ are deterministic and the $\tilde{L}^{1}$ integral in the exponent is a deterministically scaled Lévy process, it is an additive process. Since $\tilde{L}^{1}$ and $\tilde{L}^{2}$ are independent, the sum is also an additive process such that $F(t, T)$ is exponential additive.

Expressing the future process in terms of the processes $\tilde{L}^{1}$ and $\tilde{L}^{2}$ that are martingales turns out to be useful for estimation purposes. However, one can equally well express the future based on the original processes and the above formula holds with $\tilde{L}^{i}$ and $\tilde{\Psi}_{i}^{\theta}$ replaced by $L^{i}$ and $\Psi_{2}^{\theta}$ 
and $\frac{\partial}{\partial z} \Psi_{i}^{\theta}(0)$ set to zero for $i \in\{1,2\}$. We denote the deterministic factors obtained then by $h_{1}(T)$ and $h_{2}(T-t)$.

We are actually not interested in the future that delivers at a fixed point in time but in the future delivering continuously over a period $\left[T_{1}, T_{2}\right]$. Using (5.1) we find that

$$
F\left(t, T_{1}, T_{2}\right)=\frac{1}{T_{2}-T_{1}} \int_{T_{1}}^{T_{2}} h_{1}(T) h_{2}(T-t) \exp \left(\tilde{L}_{t}^{2}+e^{-\lambda T} \int_{0}^{t} e^{\lambda u} \mathrm{~d} \tilde{L}_{u}^{1}\right) \mathrm{d} T
$$

for $t<T_{1}$. In contrast to $F(t, T)$, the process $F\left(t, T_{1}, T_{2}\right)$ is not of exponential additive type due to the $T$ dependency in the scaling of the $\tilde{L}^{1}$ integral. For that reason we shall aim at finding scaling terms $\Sigma^{T_{1}, T_{2}}(t)=\left(\Sigma_{1}(t), \Sigma_{2}(t)\right)$ such that

$$
F\left(t, T_{1}, T_{2}\right) \approx F\left(0, T_{1}, T_{2}\right) \exp \left(-\int_{0}^{t} \Psi^{\theta}\left(\left(\Sigma_{1}(s), \Sigma_{2}(s)\right) \mathrm{d} s+\int_{0}^{t} \Sigma^{T_{1}, T_{2}}(s) \cdot \mathrm{d} L_{s}\right)\right.
$$

We will show in the next subsection how to find $\Sigma^{T_{1}, T_{2}}(t)$ by moment matching techniques.

\subsection{Matching $\Sigma^{T_{1}, T_{2}}(t)$}

We now aim at finding a scaling term $\Sigma^{T_{1}, T_{2}}(t)=\left(\Sigma_{1}(t), \Sigma_{2}(t)\right)$ that approximates the dynamics (5.5) sufficiently well, but is such that the resulting model is of exponential additive type. We shall match first and second moments as suggested and well performing in (Benth, 2010) for the Geometric Brownian motion case.

We denote by $\hat{F}\left(t, T_{1}, T_{2}\right)$ the approximating process given by the righ-hand side of 5.5 ). The first moment determines $\hat{F}\left(0, T_{1}, T_{2}\right)$ due to the martingale property and calculates using (5.1) as

$$
\hat{F}\left(0, T_{1}, T_{2}\right)=\frac{1}{\left(T_{2}-T_{1}\right)} \int_{T_{1}}^{T_{2}} h_{1}(T) h_{2}(T) \mathrm{d} T
$$

with $h_{1}(T)$ and $h_{2}(T)$ defined after Proposition 5.1. We calculate the second moment of $F\left(T_{2}, T_{1}, T_{2}\right)$ using integration by parts as

$$
\begin{aligned}
& \mathbb{E}^{\mathbb{Q}_{\theta}}\left[\frac{1}{\left(T_{2}-T_{1}\right)^{2}}\left(\int_{T_{1}}^{T_{2}} S_{T} \mathrm{~d} T\right)^{2}\right]=\frac{2}{\left(T_{2}-T_{1}\right)^{2}} \int_{T_{1}}^{T_{2}} \int_{T_{1}}^{u} \mathbb{E}^{\mathbb{Q}_{\theta}}\left[S_{u} S_{T}\right] \mathrm{d} T \mathrm{~d} u \\
= & \frac{2}{\left(T_{2}-T_{1}\right)^{2}} \int_{T_{1}}^{T_{2}} \int_{T_{1}}^{u} S_{0}^{2} h_{1}(u) h_{1}(T) e^{\int_{0}^{u} \Psi^{\theta}\left(e^{\lambda(s-u)}+e^{\lambda(s-T)}, 2\right) \mathrm{d} s+\int_{u}^{T} \Psi^{\theta}\left(e^{\lambda(s-T)}, 1\right) \mathrm{d} s} \mathrm{~d} T \mathrm{~d} u(5.6)
\end{aligned}
$$

and for $F\left(t, T_{1}, T_{2}\right)$ as

$$
\begin{aligned}
& \mathbb{E}^{\mathbb{Q}_{\theta}}\left[\frac{1}{\left(T_{2}-T_{1}\right)^{2}}\left(\int_{T_{1}}^{T_{2}} F(t, T) \mathrm{d} T\right)^{2}\right]=\frac{2}{\left(T_{2}-T_{1}\right)^{2}} \int_{T_{1}}^{T_{2}} \int_{T_{1}}^{u} \mathbb{E}^{\theta}[F(t, u) F(t, T)] \mathrm{d} T \mathrm{~d} u \\
= & \frac{2}{\left(T_{2}-T_{1}\right)^{2}} \int_{T_{1}}^{T_{2}} \int_{T_{1}}^{u} S_{0}^{2} h_{1}(u) h_{1}(T) e^{h(t, u, T)} \mathrm{d} T \mathrm{~d} u
\end{aligned}
$$

with $h(t, u, T)$ given by

$$
h(t, u, T)=\int_{0}^{t} \Psi^{\theta}\left(e^{\lambda(s-u)}+e^{\lambda(s-T)}, 2\right) \mathrm{d} s+\int_{t}^{u} \Psi^{\theta}\left(e^{\lambda(s-u)}, 1\right) \mathrm{d} s+\int_{t}^{T} \Psi^{\theta}\left(e^{\lambda(s-T)}, 1\right) \mathrm{d} s .
$$


On the other hand, the second moments of $\hat{F}\left(t, T_{1}, T_{2}\right)$ compute as

$$
\mathbb{E}^{\mathbb{Q}_{\theta}}\left[\hat{F}\left(t, T_{1}, T_{2}\right)^{2}\right]=\hat{F}\left(0, T_{1}, T_{2}\right)^{2} e^{-2 \int_{0}^{t} \Psi^{\theta}\left(\Sigma^{T_{1}, T_{2}}(s)\right) \mathrm{d} s+\int_{0}^{t} \Psi^{\theta}\left(2 \Sigma^{T_{1}, T_{2}}(s)\right) \mathrm{d} s}
$$

Equating the two right hand sides of (5.7) and (5.8), taking logarithm and differentiating with respect to $t$ we obtain

$$
-2 \Psi^{\theta}\left(\Sigma^{T_{1}, T_{2}}(t)\right)+\Psi^{\theta}\left(2 \Sigma^{T_{1}, T_{2}}(t)\right)=\zeta\left(t, \frac{\partial h(t, u, T)}{\partial t}\right) / \zeta(t, 1)
$$

with $\zeta(t, \eta(t, u, T))$ defined for sufficiently regular and integrable functions $\eta$ by

$$
\zeta(t, \eta(t, u, T)):=\frac{2}{\left(T_{2}-T_{1}\right)^{2}} \int_{T_{1}}^{T_{2}} \int_{T_{1}}^{u} S_{0}^{2} h_{1}(u) h_{1}(T) e^{h(t, u, T)} \eta(t, u, T) \mathrm{d} T \mathrm{~d} u .
$$

The integral with respect to $L^{2}$ has no dependency on the maturity of the future and can be taken out of (5.4). It is therefore unaffected by the integration with respect to $T$ and it appears reasonable to chose $\Sigma_{2}(t)=1$. Then, the moment condition simplifies, since the cumulant function for the independent processes $L^{1}$ and $L^{2}$ add up, to

$$
-2 \Psi_{1}^{\theta}\left(\Sigma_{1}(t)\right)+\Psi_{1}^{\theta}\left(2 \Sigma_{1}(t)\right)=\zeta\left(t, \frac{\partial h_{1}(t, u, T)}{\partial t}\right) / \zeta(t, 1)
$$

with $\frac{\partial h_{1}(t, u, T)}{\partial t}:=\Psi_{1}^{\theta}\left(e^{\lambda(t-u)}+e^{\lambda(t-T)}\right)-\Psi_{1}^{\theta}\left(e^{\lambda(t-u)}\right)-\Psi_{1}^{\theta}\left(e^{\lambda(t-T)}\right)$ being the $\Psi_{1}^{\theta}$ contribution of $\frac{\partial h(t, u, T)}{\partial t}$.

In the following we restrict to the situation where $L^{i}$ are Normal Inverse Gaussian distributed Lévy processes, a choice motivated by the empirical analysis to come. The cumulant transform for the NIG distribution is given by

$$
\Psi_{\mathrm{NIG}}(z)=\delta\left\{\sqrt{\alpha^{2}-\beta^{2}}-\sqrt{\alpha^{2}-(\beta+z)^{2}}\right\}+\mu z,
$$

(see (Barndorff-Nielsen, 1998)). To show that there exists a unique choice $\Sigma_{1}(t)$ that satisfies (5.11) we need the following Lemma.

Lemma 5.2. The function $\Psi_{N I G}(z)$ is strictly super additive, i.e. $\Psi_{N I G}(x+y)>\Psi_{N I G}(x)+$ $\Psi_{N I G}(y)$, for $x, y \in \mathbb{R}_{+}, x+y \leq \alpha-\beta$ and the function $\Psi_{N I G}(2 z)-2 \Psi_{N I G}(z)$ is strictly increasing in $z$ for $z \in \mathbb{R}, z \leq(\alpha-\beta) / 2$.

Proof. See Appendix

We assume that $\Psi^{\theta}(z)=\Psi_{1}^{\theta}\left(z_{1}\right)+\Psi_{2}^{\theta}\left(z_{2}\right) \in \mathbb{R}$ for $z=\left(z_{1}, z_{2}\right) \in[0,2] \times[0,2]$. Due to the last Lemma, both sides of Equation (5.11) are positive and, since $\Psi_{\mathrm{NIG}}(2 z)-2 \Psi_{\mathrm{NIG}}(z)$ is strictly increasing, there exists a unique choice $\Sigma_{1}(t)$ satisfying (5.11) or more generally there exists $\Sigma^{T_{1}, T_{2}}(t)$ satisfying $(5.9)$ for $t \leq T_{1}$.

To see that there exists a continuation $\Sigma^{T_{1}, T_{2}}(t)$, for $T_{1} \leq t \leq T_{2}$, such that $\mathbb{E}^{\theta}\left[\hat{F}\left(T_{2}, T_{1}, T_{2}\right)^{2}\right]$ equals (5.6), we notice that the integrand in (5.6) differs from the integrand appearing in $\mathbb{E}^{\theta}\left[\hat{F}\left(T_{1}, T_{1}, T_{2}\right)^{2}\right]$ by the factor

$$
e^{\int_{T_{1}}^{u} \Psi^{\theta}\left(e^{\lambda(s-u)}+e^{\lambda(s-T)}, 2\right) \mathrm{d} s-\int_{T_{1}}^{u} \Psi^{\theta}\left(e^{\lambda(s-u)}, 1\right) \mathrm{d} s-\int_{T_{1}}^{u} \Psi^{\theta}\left(e^{\lambda(s-T)}, 1\right) \mathrm{d} s},
$$

which has strictly positive exponent by Lemma 5.2 , bounded by $\left(T_{2}-T_{1}\right) \Psi^{\theta}(2,2)-2\left(T_{2}-\right.$ $\left.T_{1}\right) \Psi^{\theta}(1,1)$, such that $\Sigma^{T_{1}, T_{2}}(t)$, for $T_{1} \leq t \leq T_{2}$ can be chosen to match 5.6. However, this choice is not unique. 

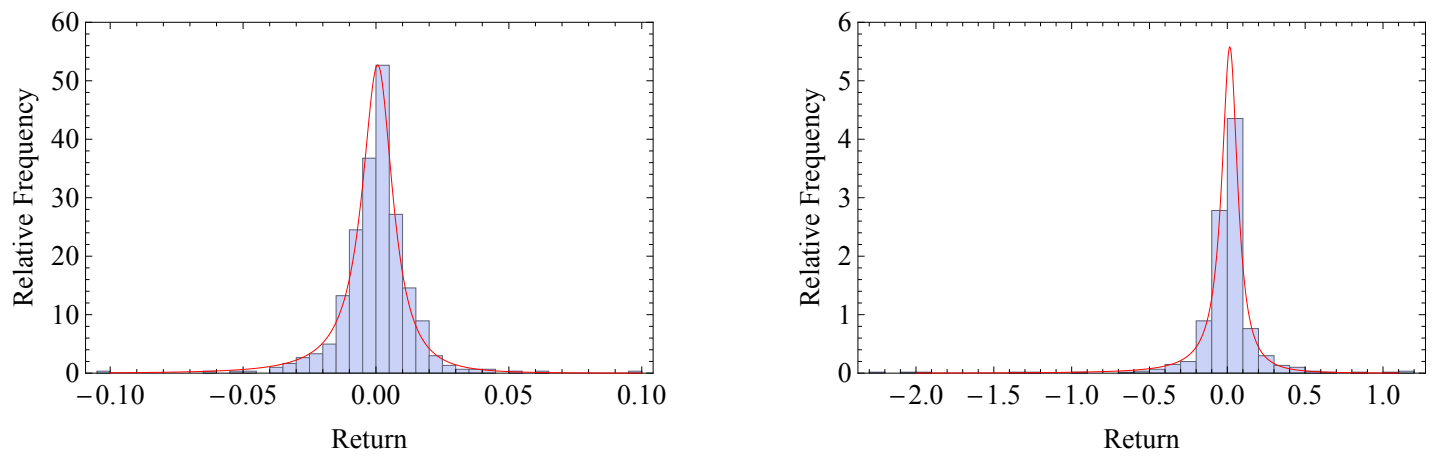

Figure 2: Left: Histogram of the empirical distribution of $L_{t}^{2}$ together with the density of the fitted NIG distribution Right: Histogram of the empirical distribution of $L_{t}^{1}$ together with the density of the fitted NIG distribution.

\subsection{Parameter estimation}

In this section we estimate the parameters for the model described in the last section based on spot and future prices on peak load electricity traded at Nord Pool. Our estimation procedure is based on the ideas of (Schwartz and Smith, 2000$)$ in jointly using future prices and spot prices to determine the parameters of the models and later extended to Lévy processes by (Benth et al. 2014) (see also (Benth and Schmeck, 2014+)). We have available average daily system power spot prices (including weekends and holidays) for the time period from 1st January 2011 until 31th May 2013, a total of 604 observations 7 Additionally we have weekday prices for a rolling series of front month and 2nd month future contracts with one month delivery period 8

We shall first determine the distribution of the long term factor by fitting a normal inverse Gaussian(NIG) distribution to the daily log returns of the future series. The NIG distribution has four parameters to estimate, the tail heaviness $\alpha$, the skewness $\beta$, the location $\mu$ and the scale parameter $\delta$. For details on the NIG distribution and its applications to finance see (Barndorff-Nielsen, 1998). Due to the exponential decay of the short term factor, as seen in the future dynamics (5.4), the future price is only driven by $L_{t}^{2}$ for $T_{1}-t \gg 0$. As will later be seen, the analysis of the spot prices suggests that the mean reversion $\lambda$ is high enough such that the impact of the short term factor $L_{t}^{1}$ is negligible for $T_{1}-t$ greater than one month. Therefore we use the second month future series for fitting $L_{t}^{2} 9^{9}$ Note that for $T_{1}-t \gg 0$ we

\footnotetext{
${ }^{7}$ Price history as shown on Bloomberg page "ENOSOSPK Index".

${ }^{8}$ Price history shown on Bloomberg page "NEP1M Comdty" and "NEP2M Comdty", respectively.

${ }^{9}$ The second month future price history as shown on Bloomberg page "NEP2M Comdty" is based on a rolling sequence of future contracts with delivery between one and two month ahead. For that reason every end of month the future contract previously shown becomes front month contract and is included in the price history "NEP1M Comdty". From that day on for "NEP2M Comdty", the next contract maturing one month later is chosen. Due to this construction is it likely that there is greater price change due to the new delivery period of
} 

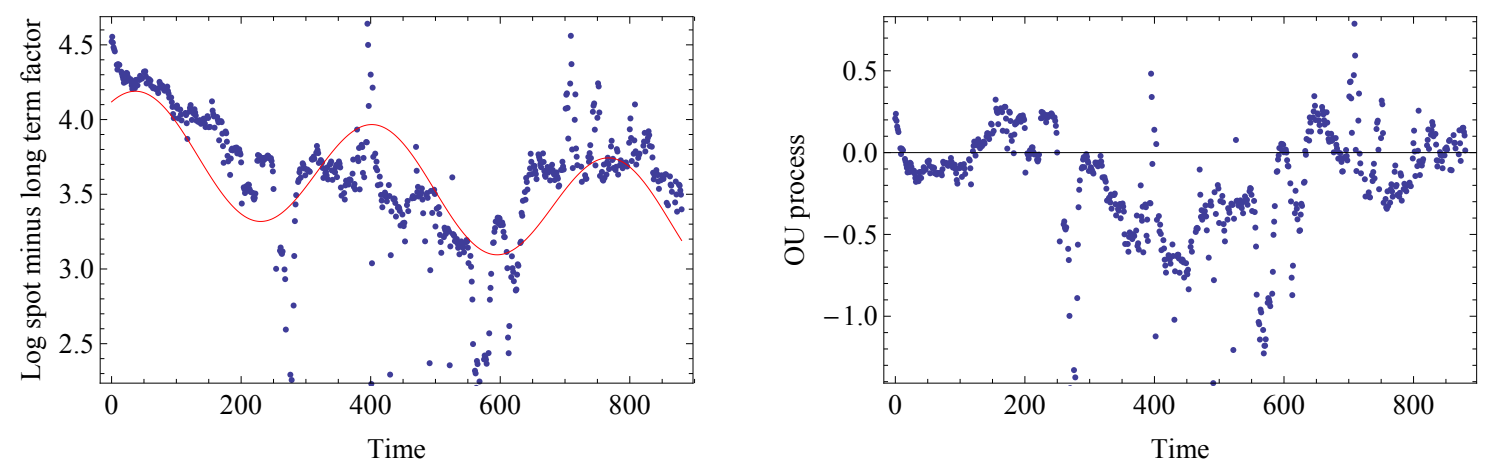

Figure 3: Left: Residual log spot price $\Lambda_{t}+X_{t}$ after subtracting long term factor $Y_{t}$, together with the fitted seasonality function(red) Right: De-seasonalized process $X_{t}$.

find $\ln (F(t, T)) \approx\left(\tilde{\Psi}_{2}^{\theta_{2}}(1)-\frac{\partial}{\partial z} \Psi_{i}^{\theta_{2}}(0)\right) t+C+L_{t}^{2}$ for some constant $C$ and the same applies to the future that delivers over time ${ }^{10}$ We can assume that $L_{t}^{2}$ is a $\mathbb{P}$ martingale by eventually subtracting its expectation and adjusting $\Lambda_{t}$ accordingly. This allows us to perform a linear regression on the log future price difference for an approximation of $\tilde{\Psi}_{2}^{\theta_{2}}(1)-\frac{\partial}{\partial z} \Psi_{i}^{\theta}(0)$ and $C$. We use this estimate to extract a time series for $L_{t}^{2}$. In Table 1 we show the estimated parameters for the normal inverse Gaussian distribution using maximum-likelihood. As a starting value for the optimization we use the parameters obtained by equating sample mean, variance, skewness and kurtosis against its theoretical counterparts. This reduces the risk of ending up in a local maximum. The empirical log returns of the future prices is shown together with the density of the estimated normal inverse Gaussian distribution in Figure 2(left). The NIG process seems to fit the distribution rather well. For an estimate of $\theta_{2}$ we impose the martingale condition, which implies that $\tilde{\Psi}_{2}^{\theta_{2}}(1)-\frac{\partial}{\partial z} \Psi_{i}^{\theta}(0)$ equals the estimated slope parameter and solve for $\theta_{2}$, which yields an estimate of -8.8196 . The negative value for $\theta_{2}$ indicates that market participants assign a slightly higher probability to negative jumps than assigned under the physical measure $\mathbb{P}$, which appears market-reasonable, as this implies a negative risk premium in the long end.

\begin{tabular}{r|rrrr} 
& $\alpha$ & $\beta$ & $\mu$ & $\delta$ \\
\hline$L_{t}^{2}$ & 1.9240 & -0.8860 & 0.0176 & 0.0622 \\
$L_{t}^{1}$ & 33.3008 & -1.0988 & -0.0009 & 0.0071 \\
\hline
\end{tabular}

Table 1: Estimated NIG parameters for $L_{t}^{1}$ and $L_{t}^{2}$

the current future contract, because the average seasonality in the spot price might be different. To capture only the stochastic factor, we calculate the log return for the first trading day of the month from the price change between the last day of the month in the series "NEP2M Comdty" and the first day of the next month in the series "NEP1M Comdty".

${ }^{10}$ Alternatively one could directly look at the deterministic part of the logarithm of $\hat{F}\left(t, T_{1}, T_{2}\right)$ for $T_{1}-t \gg 0$. 
To estimate the second factor we use the logarithm of the spot history. First, we subtract the realization of $L_{t}^{2}$. On the residual price history we fit a seasonality function. To cover yearly effects due to seasonality in supply and demand, we fit a sinus function with yearly frequency. Since we imposed the martingale condition on $L_{t}^{2}$, we add a linear term to compensate for eventually having artificially added a linear term to $L_{t}^{2}$. Thus we estimate the parameters of the function

$$
b_{1}+b_{2} \sin \left[2 \pi\left(t / 365-b_{3}\right)\right]+b_{4} t .
$$

The estimated parameters are shown in Table 2. The residual log spot price $\Lambda_{t}+Y_{0}+X_{t}$, together with its fitted function $\Lambda_{t}$ is plotted in Figure 3 (left). For a rough estimate of $Y_{0}$ we use the difference between log future price on the first day and $\Lambda_{0}$. Figure 3 (right) shows the residual returns $X_{t}$. To fit an Ornstein-Uhlenbeck process to the observed residuals we regress the observed $X_{t+1}$ on $X_{t}$. This gives us a slope of 0.8367 , resulting in $\lambda=0.1783$ per day, corresponding to a half-life of 4 days (see (Benth et al. 2008$)$ for a definition of half-life). With this rather fast reversion speed, our choice to neglect the short term factor in the second month future series appears reasonable. Having estimated $\lambda$ now allows us to retrieve a history for $L^{1}$. The NIG parameter estimates for $L^{1}$ are shown in Table 1 and the fitted NIG distribution together with the empirical density is shown in Figure 2(right). The sample correlation of $L^{1}$ and $L^{2}$ is very low with $2.92 \%$, which shows that our assumption of using two independent Lévy processes is reasonable. We are not able to retrieve $\theta_{1}$ and chose $\theta_{1}=0$. To determine the market implied value for $\theta_{1}$ we would have to take the short term future prices into account. Our model can therefore be considered as calibrated only to long term future contracts.

\begin{tabular}{rrrr}
$b_{1}$ & $b_{2}$ & $b_{3}$ & $b_{4}$ \\
\hline 3.8354 & 0.3783 & -0.8655 & -0.0006 \\
\hline
\end{tabular}

Table 2: Estimated parameters for the seasonality function $\Lambda_{t}$

Using the procedures described in Subsection 5.1 we derive the scaling term $\Sigma^{T_{1}, T_{2}}(t)$ for an approximating model that is exponential additive. The result of this moment matching procedure is shown in Figure 4(upper left), where we show both, the scaling term for the long term factor $L^{2}$ and the short term factor $L^{1}$. It turns out that due to the high level of mean reversion for the short term factor, the dynamics is only influenced by this factor shortly before the delivery period starts, and, due to the averaging, is significantly reduced compared to the artificial future delivering at time point $T_{1}$. In Figure 4(upper right) we depict realizations of the future price. The initial price is 73.82 , which is very close to the observed price of 74 as traded on the first day of our price history, although we only account for the risk premium with respect to the factor $L^{2}$. Realizations of the spot price is presented in Figure 4 ( lower left). One can observe that it is much more volatile than the future price process due to the very volatile short term factor.

\subsection{Pricing and hedging options}

We perform a simulation of the hedge error for an example payoff. Based on the model estimates in the last subsection we calculate the option price and hedge positions for the Asian call payoff

$$
\left(\frac{1}{T_{2}-T_{1}} \int_{T_{1}}^{T_{2}} S_{r} \mathrm{~d} r-K\right)^{+}
$$

with $T_{1}=60$ and $T_{2}=90$ days and at the money strike $K=73.82$. The initial option value is 3.74 , calculated according to Equation (3.34). We simulate paths for both, the approximate 

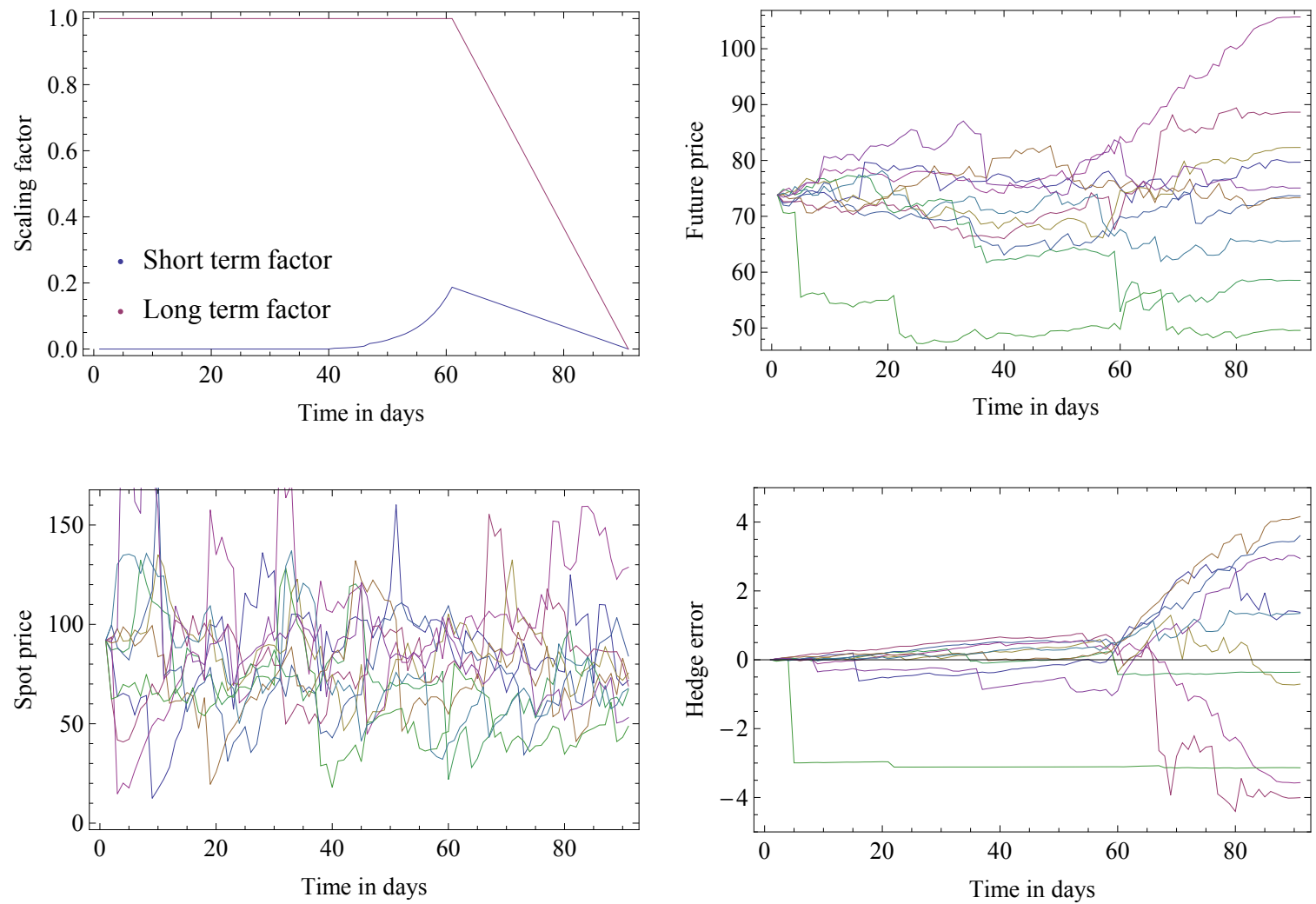

Figure 4: Upper left: Fitted function $\Sigma^{T_{1}, T_{2}}(t)$ derived by matching second moments for $t \in$ $\left[0, T_{1}\right]$ and $T_{2}$. Upper right: Sample path from the resulting exponential Lévy model. Lower left: Spot path. Lower right: Hedge error over life time of the option. 

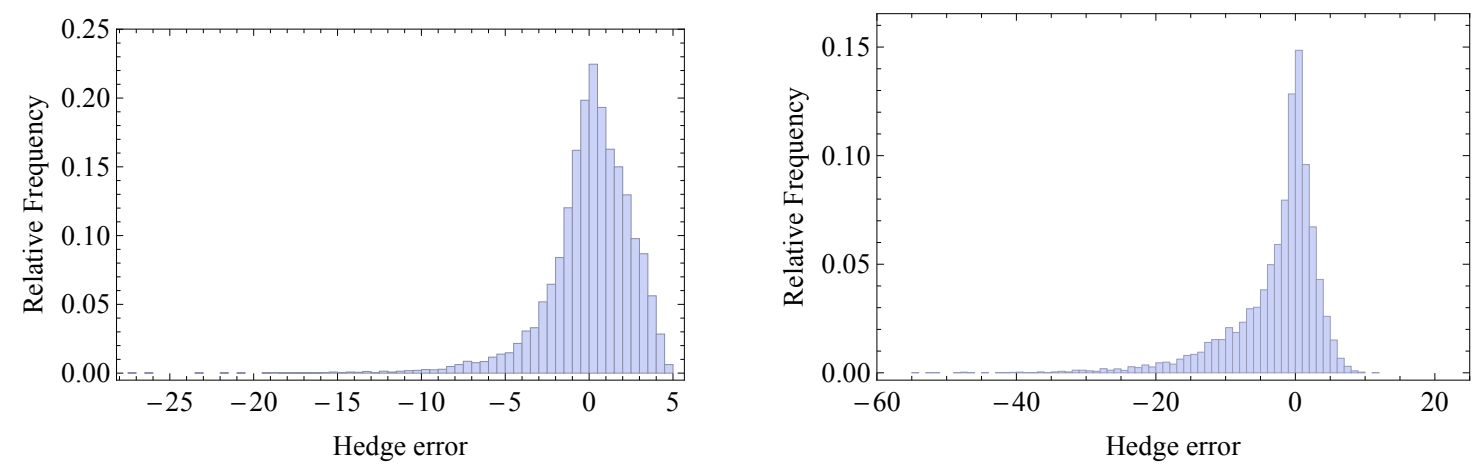

Figure 5: Left: Hedge error, measured as difference between the hedge portfolio and $\left(\hat{F}\left(T_{2}, T_{1}, T_{2}\right)-K\right)^{+}$. Right: Hedge error, measured as difference between the hedge portfolio and the true option payout.

future price process $\hat{F}\left(t, T_{1}, T_{2}\right)$ and the spot price process $S_{t}$ based on the same realizations of the process $\left(L^{1}, L^{2}\right)$ and calculate the hedge positions for $t<T_{1}$ with the integral formula (3.35) and for $T_{1}$ with the integral formula in (3.40). We assume daily rehedging in the period before $T_{1}$. In Figure 4(lower right) we show the hedge error as evolving over time for some example paths. One can see that the hedge error increases heavily during the period after $T_{1}$, when the hedge positions can not be adjusted anymore. We simulate 10000 paths and calculate the final value of the hedge portfolio. In Figure 5 (left) we show the distribution of the difference between the final value of the hedge portfolio and $\left(\hat{F}\left(T_{2}, T_{1}, T_{2}\right)-K\right)^{+}$. In Figure 5 (right) we show the difference of the hedge portfolio and the true option payout based on the average stock price in $\left[T_{1}, T_{2}\right]$ as described in Equation (5.15). It can be seen that this histogram is more widespread, which is not surprising since the process $\hat{F}\left(T_{2}, T_{1}, T_{2}\right)$ is only an approximation to the dynamics of the average stock price and the hedge positions calculated in this model are not necessarily optimal. The square root of the expected squared hedge difference is relatively high with 2.37 and 7.51 respectively, which makes clear that the trading restriction in the delivery period significantly increases the risk when using the future for hedging.

\section{A Appendix: Counterexample for Proposition 2.6 when $X$ is not a local martingale}

To see that Proposition 2.6 can not be generalized to allow $X$ to have a drift $(\alpha \neq 0)$, we consider an explicit counter example. Let $X$ have the form

$$
X_{t}=B_{t}+\int_{0}^{t} \mathbf{1}_{\{s \leq \widehat{T}\}} \mathrm{d}[B, B]_{s},
$$


with $T_{1}<\widehat{T}<T_{2}$ and $B$ a Brownian motion. The process $X$ clearly fulfills the required assumptions such that Proposition 2.4 provides a unique solution for each square integrable payoff, given some initial portfolio value. We consider the claim $X_{\widehat{T}}$. Without the trading restriction it can be perfectly hedged with an initial capital of $X_{0}$ by the strategy $X_{0}+\int_{0}^{T_{2}} \mathbf{1}_{\{s \leq \widehat{T}\}} \mathrm{d} X_{s}=$ $X_{0}+\int_{0}^{\widehat{T}} 1 \mathrm{~d} X_{s}$. Since $X$ is not a martingale, the two spaces $\mathcal{N}_{\theta_{1}, X}$ and $\mathcal{N}_{\theta_{2}, X}$ are not orthogonal. To see that they are still direct, let $x \in \mathcal{N}_{\theta_{1}, X} \cap \mathcal{N}_{\theta_{2}, X}$. Then $x=\int_{0}^{T_{1}} \psi_{s} \mathrm{~d} X_{s}=\widehat{\psi}\left(X_{T_{2}}-X_{T_{1}}\right)$ for some process $\left(\psi_{t}\right)_{0<t \leq T_{1}}$ and $\mathcal{F}_{T_{1}}$-measurable $\widehat{\psi}$. However, by the norm-equivalence used in the proof of Lemma 2.3 one can easily see that also $\int_{0}^{T_{1}} \psi_{s} \mathrm{~d} B_{s}=\widehat{\psi}\left(B_{T_{2}}-B_{T_{1}}\right)$, which implies that $x=0$ by orthogonality of $\int_{0}^{T_{1}} \psi_{s} \mathrm{~d} B_{s}$ and $\widehat{\psi}\left(B_{T_{2}}-B_{T_{1}}\right)$. We have to find out what happens to $\int_{0}^{\widehat{T}} 1 \mathrm{~d} X_{s}=\int_{0}^{T_{1}} 1 \mathrm{~d} X_{s}+\int_{T_{1}}^{\widehat{T}} 1 \mathrm{~d} X_{s}$ when projected onto $\mathcal{N}_{\theta_{1}, X} \oplus \mathcal{N}_{\theta_{2}, X}$. By linearity of the projection, the fact that $\mathcal{N}_{\theta_{1}, X}$ and $\mathcal{N}_{\theta_{2}, X}$ are direct and that $\int_{0}^{T_{1}} 1 \mathrm{~d} X_{s}$ is in $\mathcal{N}_{\theta_{1}, X}$ we get that $\Pi^{\mathcal{N}_{\theta_{1}, X} \oplus \mathcal{N}_{\theta_{2}, X}}\left(\int_{0}^{\widehat{T}} 1 \mathrm{~d} X_{s}\right)=\int_{0}^{T_{1}} 1 \mathrm{~d} X_{s}+\Pi^{\mathcal{N}_{\theta_{1}, X} \oplus \mathcal{N}_{\theta_{2}, X}}\left(\int_{T_{1}}^{\widehat{T}} 1 \mathrm{~d} X_{s}\right)$. It therefore remains to analyze $\Pi^{\mathcal{N}_{\theta_{1}, X} \oplus \mathcal{N}_{\theta_{2}, X}}\left(\int_{T_{1}}^{\widehat{T}} 1 \mathrm{~d} X_{s}\right)$.

We shall directly approximate $\int_{T_{1}}^{\widehat{T}} 1 \mathrm{~d} X_{s}=X_{\widehat{T}}-X_{T_{1}}$ with elements in $\mathcal{N}_{\theta_{2}, X}$ and observe then that one can do better by approximating with elements in $\mathcal{N}_{\theta_{1}, X} \oplus \mathcal{N}_{\theta_{2}, X}$. We chose $T_{1}=1, \widehat{T}=2$ and $T_{2}=3$. By definition of the process $X$ and properties of the Brownian motion we obtain $N:=X_{2}-X_{1} \sim N(1,1)$, where $N\left(\mu, \sigma^{2}\right)$ denotes the normal distribution with mean $\mu$ and standard deviation $\sigma$. Due to the independent increment property of Brownian motion is suffices to minimize $\mathbb{E}\left[\left(N-a\left(X_{3}-X_{1}\right)\right)^{2}\right]$ with some deterministic $a \in \mathbb{R}$. For given $a$, the trading gain $a\left(X_{3}-X_{1}\right)$ equals $a N+a M$ with $M \sim N(0,1)$ by the definition of $X$ and the hedge error is $\epsilon(a):=(1-a) N-a M \sim N\left(1-a,(1-a)^{2}+a^{2}\right)$. Further $\mathbb{E}\left[\epsilon(a)^{2}\right]=2(1-a)^{2}+a^{2}$ by properties of the normal distribution. This is minimized by choosing $a$ equal to $2 / 3$, resulting in a squared error of $\mathbb{E}\left[\epsilon(2 / 3)^{2}\right]=2 / 3$.

We now show that this approximation can be improved by approximating with elements in $\mathcal{N}_{\theta_{1}, X} \oplus \mathcal{N}_{\theta_{2}, X}$. For $b \in \mathbb{R}$ we get $b\left(X_{1}-X_{0}\right) \sim N\left(b, b^{2}\right)$ and $b\left(X_{1}-X_{0}\right) \in \mathcal{N}_{\theta_{1}, X}$. Clearly then $b\left(X_{1}-X_{0}\right)-a\left(X_{3}-X_{1}\right) \in \mathcal{N}_{\theta_{1}, X} \oplus \mathcal{N}_{\theta_{2}, X}$ and one can see that $\epsilon(a, b):=N-b\left(X_{1}-X_{0}\right)-a\left(X_{3}-\right.$ $\left.X_{1}\right) \sim N\left(1-a-b,(1-a)^{2}+a^{2}+b^{2}\right)$ and $\mathbb{E}\left[\epsilon(a, b)^{2}\right]=(1-a-b)^{2}+(1-a)^{2}+a^{2}+b^{2}$. Choosing $a=0.6$ and $b=0.2$, gives $\mathbb{E}\left[\epsilon(a, b)^{2}\right]=0.6<(2 / 3)$, which shows that $\Pi^{\mathcal{N}_{\theta_{1}, X} \oplus \mathcal{N}_{\theta_{2}}, X}\left(\int_{T_{1}}^{\widehat{T}} 1 \mathrm{~d} X_{s}\right) \notin$ $\mathcal{N}_{\theta_{2}, X}$.

\section{B Appendix: Proofs}

Proof of Lemma 3.2. We calculate $\mathcal{L}\left\{c_{2}(\cdot)\right\}(z)$ for $z$ with $\Re(z)>2$. The others can be calculated similarly. By definition the Laplace transform $\mathcal{L}\left\{c_{2}(\cdot)\right\}(z)$ defined for $z \in \mathbb{C}$ calculates as

$$
\begin{aligned}
\mathcal{L}\left\{c_{2}(\cdot)\right\}(z) & =\int_{-\infty}^{\infty} e^{-z y} c_{2}(y) \mathrm{d} y \\
& =\int_{-\infty}^{\infty} e^{-z y}\left(X_{T_{1}} e^{y}-K\right)^{+}\left(X_{T_{1}} e^{y}-X_{T_{1}}\right) \mathrm{d} y
\end{aligned}
$$


Since the integrand becomes zero for $y \leq \ln \left(\frac{K}{X_{T_{1}}}\right)$, the expression simplifies to

$$
\begin{aligned}
\mathcal{L}\left\{c_{2}(\cdot)\right\}(z) & =\int_{\ln \left(\frac{K}{X_{T_{1}}}\right)}^{\infty} e^{-z y}\left(X_{T_{1}} e^{y}-K\right)\left(X_{T_{1}} e^{y}-X_{T_{1}}\right) \mathrm{d} y \\
& =\int_{\ln \left(\frac{K}{X_{T_{1}}}\right)}^{\infty} X_{T_{1}}^{2} e^{(2-z) y}-\left(K X_{T_{1}}+X_{T_{1}}^{2}\right) e^{(1-z) y}+K X_{T_{1}} e^{-z y} \mathrm{~d} y .
\end{aligned}
$$

The integral converges for $z \in \mathbb{C}$ with $\Re(z)>2$ and becomes

$$
\begin{aligned}
\mathcal{L}\left\{c_{2}(\cdot)\right\}(z) & =\left[\frac{X_{T_{1}}^{2}}{2-z} e^{(2-z) y}-\frac{\left(K X_{T_{1}}+X_{T_{1}}^{2}\right)}{1-z} e^{(1-z) y}-\frac{K X_{T_{1}}}{z} e^{-z y}\right]_{\ln \left(\frac{K}{X_{T_{1}}}\right)}^{\infty} \\
& =-\frac{X_{T_{1}}^{2}}{2-z}\left(\frac{K}{X_{T_{1}}}\right)^{(2-z)}+\frac{\left(K X_{T_{1}}+X_{T_{1}}^{2}\right)}{1-z}\left(\frac{K}{X_{T_{1}}}\right)^{(1-z)}+\frac{K X_{T_{1}}}{z}\left(\frac{K}{X_{T_{1}}}\right)^{-z}
\end{aligned}
$$

such that $\mathcal{L}\left\{c_{2}(\cdot)\right\}(z)=L_{2}(z)$ for $\Re(z)>2$.

Proof of Lemma 3.5. Again, we condition on the filtration $\mathcal{G}_{t}$ defined in $(3.12)$ and obtain

$$
\begin{aligned}
C_{t}=\mathbb{E}\left[C\left(X_{T_{2}}\right) \mid \mathcal{F}_{t}\right] & =\mathbb{E}\left[C\left(X_{t} e^{-\frac{1}{2} \int_{t}^{T_{2}} \sigma_{s}^{2} \mathrm{~d} s+\int_{t}^{T_{2}} \sigma_{s} \mathrm{~d} B_{s}}\right) \mid \mathcal{F}_{t}\right] \\
& =\mathbb{E}\left[\mathbb{E}\left[C\left(X_{t} e^{-\frac{1}{2} \int_{t}^{T_{2}} \sigma_{s}^{2} \mathrm{~d} s+\int_{t}^{T_{2}} \sigma_{s} \mathrm{~d} B_{s}}\right) \mid \mathcal{G}_{t}\right] \mid \mathcal{F}_{t}\right] \\
& =X_{t} \mathbb{E}\left[\Phi\left(d_{1}\right) \mid \mathcal{F}_{t}\right]-K \mathbb{E}\left[\Phi\left(d_{2}\right) \mid \mathcal{F}_{t}\right]
\end{aligned}
$$

and by the same arguments

$$
P_{t}=\mathbb{E}\left[P\left(X_{T_{2}}\right) \mid \mathcal{F}_{t}\right]=K \mathbb{E}\left[\Phi\left(-d_{2}\right) \mid \mathcal{F}_{t}\right]-X_{t} \mathbb{E}\left[\Phi\left(-d_{1}\right) \mid \mathcal{F}_{t}\right]
$$

where $\Phi(x)$ is the cumulative normal distribution function and

$$
\begin{aligned}
& d_{1}=\frac{\ln \frac{X_{t}}{K}+\frac{1}{2} \int_{t}^{T_{2}} \sigma_{s}^{2} \mathrm{~d} s}{\sqrt{\int_{t}^{T_{2}} \sigma_{s}^{2} \mathrm{~d} s}} \\
& d_{2}=d_{1}-\sqrt{\int_{t}^{T_{2}} \sigma_{s}^{2} \mathrm{~d} s}
\end{aligned}
$$

Using the representation of the integrated variance 3.9 , and abbreviating $Z_{t, T_{2}}:=\int_{t}^{T_{2}} \epsilon(u, T) \mathrm{d} L_{u}$ it becomes clear that $d_{1}$ and $d_{2}$ only depend on $X_{t}, \sigma_{t}^{2}$ and $Z_{t, T_{2}}$ for $s \leq t \leq T$. Denote by $d_{1}\left(X_{t}, \sigma_{t}^{2}, Z_{t, T_{2}}\right)$ and $d_{2}\left(X_{t}, \sigma_{t}^{2}, Z_{t, T_{2}}\right)$ the functions defined in $\left.\mathrm{B} .1\right)$ where we now make explicit their dependency. This gives

$$
C\left(t, X_{t}, \sigma_{t}^{2}\right)=X_{t} \mathbb{E}\left[\Phi\left(d_{1}\left(X_{t}, \sigma_{t}^{2}, Z_{t, T_{2}}\right)\right)\right]-K \mathbb{E}\left[\Phi\left(d_{2}\left(X_{t}, \sigma_{t}^{2}, Z_{t, T_{2}}\right)\right)\right]
$$

where the conditioning vanishes due to the independence of $Z_{t, T_{2}}$ on $\mathcal{F}_{t}$.

We want to differentiate under the integral sign (see for example (Bauer, 2001, Lemma 16.2.) and we have to verify that the differentiated function is bounded by some positive integrable function on $\Omega$. This can easily be verified for the partial derivatives with respect to $X_{t}$. Slightly more involved is the partial derivative with respect to $\sigma^{2}$. We formally differentiate with respect 
to $\sigma^{2}$ and obtain

$$
\begin{aligned}
\frac{\partial C\left(t, X_{t}, \sigma_{t}^{2}\right)}{\partial \sigma_{t}^{2}}= & X_{t} \mathbb{E}\left[n\left(d_{1}\left(X_{t}, \sigma_{t}^{2}, Z_{t, T}\right)\right) \frac{\partial d_{1}\left(X_{t}, \sigma_{t}^{2}, Z_{t, T}\right)}{\partial \sigma_{t}^{2}}\right] \\
& -K \mathbb{E}\left[n\left(d_{2}\left(X_{t}, \sigma_{t}^{2}, Z_{t, T}\right)\right) \frac{\partial d_{2}\left(X_{t}, \sigma_{t}^{2}, Z_{t, T}\right)}{\partial \sigma_{t}^{2}}\right]
\end{aligned}
$$

with $n(x)$ being the density of the normal distribution. We have to verify integrability of the quantities below the expectation sign on the right hand side to justify this. We calculate

$$
\begin{aligned}
\frac{\partial d_{1}\left(X_{t}, \sigma_{t}^{2}, Z_{t, T}\right)}{\partial \sigma_{t}^{2}}= & \frac{1}{4} \epsilon(t, T)\left(\sigma_{t}^{2} \epsilon(t, T)+Z_{t, T}\right)^{-\frac{1}{2}} \\
& -\epsilon(t, T) \frac{1}{2}\left(\epsilon(t, T)+Z_{t, T}\right)^{-\frac{3}{2}} \ln \left(\frac{X_{t}}{K}\right)
\end{aligned}
$$

and

$$
\frac{\partial d_{2}\left(X_{t}, \sigma_{t}^{2}, Z_{t, T}\right)}{\partial \sigma_{t}^{2}}=\frac{\partial d_{1}\left(X_{t}, \sigma_{t}^{2}, Z_{t, T}\right)}{\partial \sigma_{t}^{2}}-\epsilon(t, T)\left(\sigma_{t}^{2} \epsilon(t, T)+Z_{t, T}\right)^{-\frac{1}{2}}
$$

with $\epsilon(t, T)$ as defined in Section 3.2. Both expressions are bounded by $D \ln X_{t}+C$ for suitable $C, D \in \mathbb{R}$ since $\sigma_{t}^{2} \geq \sigma_{0}^{2} \epsilon(0, T)$. Additionally $n(x)$ is bounded by $1 / \sqrt{2 \pi}$ such that the functions inside the expectation signs are also bounded by $D \ln X_{t}+C$, which has finite expectation under $\mathbb{Q}$ by the martingale property of $X_{t}$ and Jensens inequality. The second derivative can be treated similar. The proof for $P\left(t, X_{t}, \sigma_{t}^{2}\right)$ works the same way. It remains to show differentiability with respect to $t$. For $\sigma_{t}$ and $X_{t}$ fixed, we find that $C\left(t, X_{t}, \sigma_{t}^{2}\right)=\mathbb{E}\left[\zeta\left(\sigma_{t}^{2}, X_{t}, Z_{t, T_{2}}\right)\right]$ for a suitable function $\zeta$ by (B.3). Using Laplace inversion, stochastic Fubini and the key formula again we can calculate

$$
\begin{aligned}
\mathbb{E}\left[\zeta\left(\sigma_{t}^{2}, X_{t}, Z_{t, T_{2}}\right)\right] & =\mathbb{E}\left[\frac{1}{2 \pi i} \int_{c-i \infty}^{c+i \infty} \mathcal{L}\left\{\zeta\left(\sigma_{t}^{2}, X_{t}, \cdot\right)\right\}(z) e^{\left.z Z_{t, T_{2}} \mathrm{~d} z\right]}\right. \\
& =\frac{1}{2 \pi i} \int_{c-i \infty}^{c+i \infty} \mathcal{L}\left\{\zeta\left(\sigma_{t}^{2}, X_{t}, \cdot\right)\right\}(z) \mathbb{E}\left[e^{\left.z Z_{t, T_{2}}\right] \mathrm{d} z}\right. \\
& =\frac{1}{2 \pi i} \int_{c-i \infty}^{c+i \infty} \mathcal{L}\left\{\zeta\left(\sigma_{t}^{2}, X_{t}, \cdot\right)\right\}(z) e^{\int_{t}^{T_{2}} \kappa\left(\epsilon\left(u, T_{2}\right)\right) \mathrm{d} u} \mathrm{~d} z .
\end{aligned}
$$

Using differentiation under the integral sign in the last expression and the conditions posed on the function $\lambda_{t}$ yields differentiability.

Proof of Lemma 5.2. First note that $\Psi_{\mathrm{NIG}}(z) \in \mathbb{R}$ for $z \in \mathbb{R}_{+}$only for $x \leq \alpha-\beta$. We calculate

$$
\frac{\partial^{2} \Psi_{\mathrm{NIG}}(z)}{\partial z^{2}}=\delta\left(\frac{(\beta+x)^{2}}{\left(\alpha^{2}-(\beta+x)^{2}\right)^{3 / 2}}+\frac{1}{\sqrt{\alpha^{2}-(\beta+x)^{2}}}\right),
$$

which is strictly positive for $x<\alpha-\beta$. This implies convexity of $\Psi(x)_{\mathrm{NIG}}$. Additionally observe that $\Psi_{\mathrm{NIG}}(0)=0$. Both together yields $\Psi_{\mathrm{NIG}}(x)<x /(x+y) \Psi_{\mathrm{NIG}}(x+y)$ and $\Psi_{\mathrm{NIG}}(y)<$ $y /(x+y) \Psi_{\mathrm{NIG}}(x+y)$ by applying the convexity criterion for the endpoints 0 and $x+y$ and interior point $x /(x+y)$ and $y /(x+y)$ respectively. Adding up both inequalities yields superadditivity.

Clearly, due to super additivity $\hat{\Psi}(z):=\Psi_{\mathrm{NIG}}(2 z)-2 \Psi_{\mathrm{NIG}}(z)$ is positive. To show that it is 
strictly increasing in $z$, we calculate

$$
\frac{\partial \hat{\Psi}(z)}{\partial z}=-2 \frac{\delta(\beta+x)}{\sqrt{\alpha^{2}-(\beta+x)^{2}}}+\frac{2 \delta(\beta+2 x)}{\sqrt{\alpha^{2}-(\beta+2 x)^{2}}}
$$

and show that it is strictly positive in the interval $(0,(\alpha-\beta) / 2)$. Since the function $\frac{\partial \tilde{\Psi}(z)}{\partial z}$ is zero at $z=0$ and $\lim _{z \rightarrow \alpha-\beta} \frac{\partial \hat{\Psi}(z)}{\partial z}=\infty$, by the mean value theorem it suffices to show that there are no zeros in $(0,(\alpha-\beta) / 2)$. Zeros in (B.5) result in zeros of the polynomial $3 \alpha^{2} x^{2}+2 \alpha^{2} \beta x$ under the additional restrictions

$$
((x>-\beta / 2) \wedge(x>-\beta)) \vee((x<\beta / 2) \wedge(x<-\beta)),
$$

where $\wedge$ and $\vee$ denotes logical "and" and logical "or". However, its zeros can be explicitly found as $x_{1}=0$ and $x_{2}=-\frac{2}{3} \beta$ and $x_{2}$ does not fulfill $($ B.6). Therefore $(B .5)$ is strictly positive such that $\hat{\Psi}(z)$ is strictly increasing in $(0,(\alpha-\beta) / 2)$.

\section{References}

O. E. Barndorff-Nielsen. Processes of normal inverse Gaussian type. Finance and Stochastics, 2:41-68, 1998.

O. E. Barndorff-Nielsen and N. Shephard. Non-Gaussian Ornstein-Uhlenbeck-based models and some of their uses in financial econometrics. Journal of the Royal Statistical Society: Series B (Statistical Methodology), 63:167-241, 2001.

H. Bauer. Measure and Integration Theory. de Gruyter, 2001.

F. E. Benth, J. Benth, and S. Koekebakker. Stochastic Modeling of Electricity and Related Markets, volume 11 of Advanced series on statistical science $\&$ applied probability. World Scientific, 2008.

F. E. Benth. On forward price modelling in power markets. Alternative Investments and Strategies, R. Kiesel, M. Scherer and R. Zagst (eds.), World Scientific, pages 93-122, 2010.

F. E. Benth. The stochastic volatility model of Barndorff-Nielsen and Shephard in commodity markets. Mathematical Finance, 21(4):595-625, 2011.

F. E. Benth and M. Schmeck. Pricing futures and options in electricity markets. The interrelationships between financial and energy markets. Lecture Notes in Energy 54, S. Ramos and H. Veiga (eds.), Springer Verlag, 2014+.

F. E. Benth, C. Klüppelberg, G. Müller, and L. Vos. Futures pricing in electricity markets based on stable CARMA spot models. Energy Economics, 44:392-406, 2014.

F. E. Benth, N. Lange, and T. A. Myklebust. Pricing and Hedging Quanto Options in Energy Markets. Journal of Energy Markets, 2014+.

R. Cont and P. Tankov. Financial Modelling with Jump Processes. Chapman \& Hall/CRC, 2004 .

E. Eberlein and S. Raible. Term structure models driven by general Lévy processes. Mathematical Finance, 9(1):31-53, 1999. 
H. Föllmer and M. Schweizer. Hedging of Contingent Claims under Incomplete Information. in: M. H. Davis and R. J. Elliot (eds.), Applied Stochastic Analysis, Stochastics Monographs, Gordon and Breach, 5:389-414, 1991.

H. Föllmer and D. Sondermann. Hedging of Non-Redundant Contingent Claims. in: W. Hildenbrand and A. Mas-Colell (eds.), Contributions to Mathematical Economics, North Holland, pages 205-223, 1986.

S. Goutte, N. Oudjane, and F. Russo. Variance optimal hedging for continuous time additive processes and applications. Stochastics An International Journal of Probability and Stochastic Processes, 86(1):147-185, 2014.

S. Goutte, N. Oudjane, and F. Russo. Variance optimal hedging for discrete time processes with independent increments. Application to Electricity Markets. Journal of Computational Finance, 2014+.

J. Lucia and E. Schwartz. Electricity Prices and Power Derivatives: Evidence from the Nordic Power Exchange. Review of Derivatives Research, 5(1):5-50, 2002.

P. Monat and C. Stricker. Fermeture de $G_{T}(\Theta)$ et de $L^{2}(\mathcal{F})+G_{T}(\Theta)$. In J. Azéma, M. Yor, and P. A. Meyer, editors, Séminaire de Probabilités XXVIII, volume 1583 of Lecture Notes in Mathematics, pages 189-194. Springer Berlin Heidelberg, 1994.

E. Nicolato and E. Venardos. Option pricing in stochastic volatility models of the OrnsteinUhlenbeck type. Mathematical Finance, 13(4):445-466, 2003.

P. Protter. Stochastic Integration and Differential Equations. Springer, 2005.

W. Rudin. Real and Complex Analysis. McGraw-Hill, San Francisco, 3rd edition, 1987.

K. Sato. Lévy Processes and Infinitely Divisible Distributions. Cambridge University Press, 1999.

M. Schäl. On Quadratic Cost Criteria for Option Hedging. Mathematics of Operations Research, 19:121-131, 1994.

E. S. Schwartz and J. E. Smith. Short-term variations and long-term dynamics in commodity prices. Management Science, 46(7):893-911, July 2000.

M. Schweizer. Approximating Random Variables by Stochastic Integrals. Annals of Probability, 22:1536-1575, 1994.

M. Schweizer. On the minimal martingale measure and the Föllmer-Schweizer decomposition. Stochastic Analysis and Applications, 13:573-599, 1995a.

M. Schweizer. Variance-Optimal Hedging in Discrete Time. Mathematics of Operations Research, 20:1-32, 1995b.

M. Schweizer. Martingale densities for general asset prices. Journal of Mathematical Economics, 21(4):363-378, 1992.

J. Vecer. A new PDE approach for pricing arithmetic average Asian options. Journal of Computational Finance, 4:105-113, 2001.

J. Vecer and M. Xu. Pricing Asian options in a semimartingale model. Quantitative Finance, $4(2): 170-175,2004$. 
R. Weron. Market price of risk implied by Asian-style electricity options and futures. Energy Economics, 30(3):1098-1115, 2008.

D. V. Widder. The Laplace Transform. Princeton University Press, 1946.

K. Yosida. Functional Analysis. 6th edition, 1980. 